\title{
A Tail Fiber Engineering Platform for Improved Bacterial Transduction Based Diagnostic Reagents
}

Colin N. Lam¹, Misha G. Mehta-Kolte², Natacha Martins-Sorenson'ㄹ, Barbara Eckert², Patrick H. $\operatorname{Lin}^{1}$, Kristina Chu ${ }^{2}$, Arrash Moghaddasi ${ }^{1}$, Dylan Goldman ${ }^{1}$, Hai Nguyen $^{1}$, Ryan Chan $^{1}$, Laxmi

Nukala$^{1}$, Shawn Suko ${ }^{2}$, Brett Hanson ${ }^{1}$, Richard Yuan ${ }^{1} \&$ Kyle C. Cady ${ }^{1 *}$

${ }^{1}$ Roche Molecular Systems, Santa Clara, California, USA. ${ }^{2}$ Roche Molecular Systems, Pleasanton, California, USA.

* Correspondence should be addressed to K.C.C. (kyle.cady@roche.com) 


\title{
Supplementary methods, references, figures, tables, and
}

\section{sequences}

\author{
Supplementary Methods
}

Generation of Lysate from Packaging Lines

Overnight seed cultures of engineered P1 packaging lines were inoculated from glycerol stocks into LB Lennox supplemented with relevant antibiotics and incubated at $30^{\circ} \mathrm{C}(225 \mathrm{rpm})$. The next morning, day cultures were seeded from overnight cultures and grown to exponential growth phase at $30^{\circ} \mathrm{C}(225$ $\mathrm{rpm}$ ) in $2 \mathrm{xYT}$ media. When the cultures reached $\mathrm{OD}_{600}=1.0$, cultures were induced by temperature shift from a $30{ }^{\circ} \mathrm{C}$ to a $42{ }^{\circ} \mathrm{C}$ incubator for $90 \mathrm{~min}$ with $150 \mathrm{rpm}$ shaking. After induction, the cultures were moved to $37^{\circ} \mathrm{C}$ for $60 \mathrm{~min}$ of recovery under $150 \mathrm{rpm}$ shaking. To degrade extracellular DNA and RNA, $40 \mathrm{uL}$ of benzonase (Sigma, E1014-25KU) was added and incubated at $37^{\circ} \mathrm{C}(150 \mathrm{rpm}$ ) for $45 \mathrm{~min}$. Post recovery, un-lysed cells and cell debris were pelleted by centrifugation at 17,500 g for $20 \mathrm{~min}$.

Supernatants were passed through a $0.2 \mu \mathrm{m}$ filter (VWR, 28145-501) to further remove any remaining un-lysed cells or debris. Transduction particles in clarified lysates were further concentrated by centrifugation at $17,500 \mathrm{~g}$ for $18-20 \mathrm{~h}$ at $4{ }^{\circ} \mathrm{C}$. Pelleted transduction particles were re-suspended to desired volumetric concentration with SM buffer (DI water, $0.1 \mathrm{M} \mathrm{NaCl}^{\circ} 8 \mathrm{mM} \mathrm{MgSO}_{4}, 50 \mathrm{mM} \mathrm{Tris-} \mathrm{HCl}$ $\mathrm{pH} 7.5$ ) to make Smarticles Stocks and stored at $4{ }^{\circ} \mathrm{C}$.

\section{Transmission electron microscopy of Smarticles}

Smarticles stocks were produced in-house (as described) and sent for TEM imaging at University of Colorado Boulder using the CU Boulder Electron Microscopy Service (Boulder, Co). Briefly, $6-8 \mu \mathrm{L}$ of sample was processed by negative staining with aqueous $2 \%$ uranyl acetate stain for $30 \mathrm{sec}$. This is repeated $2-3$ times before imagining using a Tecnai T12 100-kV transmission electron microscope from FEI (Hillsboro, OR) and AMT (Woburn, MA) 2k x 2k digital camera.

\section{Construction of P1 Smarticles packaging line SEG_595}

Allelic exchange vector pRE107 (American Type Culture Collection ATCC ${ }^{\circledR} 87691^{\mathrm{TM}}$ ) was used as a backbone to remove the $\mathrm{P} 1$ terminase genes $p a c A B$ along with the packaging site embedded in the pacA gene forming plasmid p5014. Homology of $1 \mathrm{~kb}$ up and $1 \mathrm{~kb}$ downstream of the $p a c A B$ genes was amplified by PCR for Gibson assembly ${ }^{42}$ into the pRE107 backbone. Competent EC100D ${ }^{\text {TM }}$ pir-116 cells from Lucigen (Middleton, WI) were transformed per manufacturer's instruction with Gibson assembly product, and plated onto LB agar + carbenicillin. Transformed colonies were verified by PCR for insertion of P1 homology regions and sequenced. The plasmid contains the R6k origin, which requires the gene product of the pir gene for stable replication, which is contained in the EC100D ${ }^{\mathrm{TM}}$ pir-116 cells but not in the P1 host strain. The plasmid also contains the sacB gene for sucrose counter-selection of second crossover events.

The P1 host strain was made electrocompetent by taking $4 \mathrm{~mL}$ overnight culture, pelleting cells at 10,000 $\mathrm{g}$ for $1 \mathrm{~min}$ and discarding the supernatant. Remaining cell pellet was washed 3 times with $1 \mathrm{~mL}$ of icecold sterile water and suspended in $100 \mu \mathrm{L}$ of sterile water for electroporation. Cells were transformed with the pRE107- $\triangle p a c A B$ and plated onto LB + carbenicillin $100 \mathrm{ug} / \mathrm{mL}$. Resistant colonies with the plasmid 
integrated into the $\mathrm{P} 1$ genome at one of the two homology regions surrounding pacAB were then grown overnight in non-selective LB media. After growth, cultures were serially diluted onto LB (no-salt) agar + $15 \%$ sucrose for overnight counter-selection. Resultant colonies were patched onto LB agar and LB agar supplemented with carbenicillin to ensure no resistant marker was retained before both PCR and Sanger sequence verification of $p a c A B$. Strains with verified $\triangle p a c A B$ were made competent in the same manner and transformed with a pReporter plasmid to create a packaging strain for P1 based Smarticles production.

\section{Construction of pReporter plasmids}

Plasmid used as pReporter p5027 (GenBank MW263049) was constructed by PCR amplification and Gibson Assembly of $E$. coli origin from NEB sourced pUC19 (Ipswich, MA) and Pseudomonas aeruginosa origin $\mathrm{pRO} 1600$ from plasmid pUCP20 ${ }^{43}$. Pacase complex genes pacAB from P1 ATCCBAA-1001 (Manassas, VA) DNA were amplified with the native promoter. The luxAB genes, Lactococcal promoter $23 p$ and Staphylococcal terminator blaZt originated from MRSA Smarticles pReporter plasmid pSD02 (GenBank MW263053). Zeocin resistance gene was synthesized by IDT (Coralville, IA) driven by the blap promoter from pUC19 ampicillin resistance gene.

Plasmid p5028 (GenBank MW263050) is identical to p5027, but contains the aacC1 gentamicin resistance gene instead of zeocin resistance gene. Plasmid p5222 (GenBank MW263052) is identical to p5027 except for two point mutations in the luxA gene. Plasmid p5231 (GenBank MW263051) is a pReporter plasmid that uses the pBHR1 broad-host range origin (MoBiTec Göttingen, Germany), lytic replicon (oriL), pacase complex genes pacAB from Roche plasmid p2118 (Pleasanton, CA. GenBank MW263054). Reporter blapluxAB also contains luxA glow point mutations identical to p5222. The plasmid the same zeocin resistance as p5027 and p5222.

\section{Construction of TFDV plasmids}

The Tail Fiber Display Vector (TFDV) system was developed for rapid screen/expression of native or nonnative tail fibers on a bald bacteriophage P1 chassis (Table S1). Tail fiber candidates were either de novo synthesized at IDT (Coralville, IA) or identified from in-house sequenced, assembled, and annotated phages. The TFDVs were constructed using standard Gibson Assembly method per manufacturer's instructions using tail fiber PCR products and p5027 amplified by PCR. NEB5 $\alpha$ cells were transformed with the Gibson Assembly products, selected on antibiotic, correct clones identified using colony PCR, and plasmids verified by Sanger sequencing. Plasmid tail fiber region, $p a c A B$, and luxAB genes were completely sequence verified. The Bald Chassis packaging line (SEG_170) was electroporated with sequence verified TFDV constructs and selected on gentamycin.

\section{Tangerine Tail Fiber}

Plasmid p15B, a possible defective phage, contains roughly $82 \%$ DNA homology to the P1 genome ${ }^{34}$. The two differ most notably in the tail fiber region, where $\mathrm{p} 15 \mathrm{~B}$ codes for six tail fibers (Tfib) with a single tail fiber assembly protein (Tfa) gene in comparison to bacteriophage P1's native two tail fibers and two tail fiber assembly proteins. The six tail fibers have $32-35 \%$ amino acid identity to the $\mathrm{Tfa}_{\mathrm{p} 1 \mathrm{~s}}$ and $\mathrm{Tfa}_{\mathrm{p} 1 \mathrm{~S}^{\prime}}$ tail fibers, with $95 \%$ amino acid similarity in the N-terminal 250 amino acids. Sandmeir et al. have shown that expression of the $\mathrm{p} 15 \mathrm{~B}$ tail fibers in a P1 tail fiber deficient strain results in visible attached tail fibers. Each of the six different tail fiber genes in combination with the cognate tail fiber assembly protein genes were cloned into individual TFDVs to screen for functionality and reactivity. This systematic screening approach showed that Smarticles expressing p15B tail fibers are functionally able to transduce pReporter plasmid, which has not been demonstrated previously. All six tail fibers were functional resulting in transduction 
as measured by positive RLUs. This functionality highlights that the $\mathrm{N}$-terminal region is essential for attachment to the tail structure, while the C-terminal region does not need to have conserved homology to native tail fibers. This also demonstrates that the $\mathrm{C}$-terminal region is involved in host recognition and binding. The Tfib $\mathrm{p}_{\mathrm{p} 5 \mathrm{bsvo}}$ variant, labeled as "Tangerine" was reactive to a small number of strains that helped increased the E. coli detection (Supplementary Figure S4, S5, S6 and S8, Table S2). Amino acid sequences are listed in Supplemental Sequences (Sequence S13-S14).

\section{Shamrock, Jazzberry, and Orchid Tail Tiber Fusions}

Leveraging both GenBank and internal sequence databases, we were able to identify many additional diverse tail fibers for use on the chassis. We focused on sequences with large stretches of homology for fusions to diversify the C-terminal receptor binding regions. A tail fiber (GenBank AJS10005.1) from $K$. pneumoniae strain ST11 plasmid pKP12226 (GenBank KP453775.1) has high homology to the N-terminal region of $\mathrm{Tfib}_{\mathrm{P} 1 \mathrm{~S}}$ with $97 \%$ identity in the first 516 amino acids. A fusion was generated at the internal recombination site conserved on $\mathrm{Tfib}_{\mathrm{P} 1 \mathrm{~S}}$ and the $K$. pneumoniae tail fiber from a synthetic gBlock from IDT (Coralville, IA). The resulting chimeric tail fiber was labeled "Jazzberry". Interestingly, Jazzberry has high inclusivity on $E$. coli strains but only minimal reactivity on $K$. pneumoniae strains, despite the tail fiber originating from a K. pneumoniae genome. Similarly, Shigella bacteriophage sfMu also contains two tail fibers. Tfib sfMus' $_{\text {' }}$ tail fiber containing $85 \%$ identity to Tfib $_{\text {P1s' }}$ within the C-terminal 320 amino acids ${ }^{44}$. A fusion named "Shamrock" of Tfib ${ }_{\text {sfMus' }}$ with Tfib ${ }_{\mathrm{P} 1} \mathrm{~s}^{\prime}$ was generated from a synthetic gBlock from IDT (Coralville, IA). The fusion resulted in production of functional Smarticles. Functional testing of Jazzberry and Shamrock resulted in expanding inclusivity of $E$. coli strains, with a $\sim 90 \%$ theoretical inclusivity combined. Another E. coli tail fiber (GenBank WP_001000784.1) identified from E. coli strain UMNF18 plasmid pUMNF18_103 (GenBank NZ_AGTD01000005.1) contains high overall amino acid homology to Tfib $_{\text {P1S }}$ ( $85 \%$ identity), with small differences in the C-terminal region. A fusion protein, utilizing synthetic gBlock ordered from IDT (Coralville, IA), was made at the amino acid position 502 of Tfap1s and Tfib WP_001000784.1 $_{1}$ and labeled "Orchid".

These fusions have varying activity across the panels of interest (Supplementary Figure S8, Table S2). Jazzberry, Shamrock, and Orchid serve as example fusions made with high P1 homology tail fibers found in bacterial sequence databases. Amino acid sequences are listed in Supplemental Sequences (Sequence S5-8, Sequence S11-12).

\section{Banana, Rio and Gold tail fiber fusions}

The $\mathrm{N}$-terminal portion of $\mathrm{Tfib}_{\mathrm{P} 1 \mathrm{~S}}$ and $\mathrm{Tfib}_{\mathrm{P} 1 \mathrm{~S}^{\prime}}$ functions to bind to the baseplate, while the $\mathrm{C}$-terminus is responsible for binding to the target bacteria. The $\mathrm{N}$-terminal $\mathrm{S}_{\mathrm{c}}$ as defined by the cin recombination site does not serve as a structural boundary between the two regions. Lacking structural information, conserved regions and/or domain structure prediction in phylogenetically distant tail fibers were relied on. Motifs found in E. coli phage P2 and P1 ${ }^{45}$ along with a known fusion between a $P$. aeruginosa R-type pyocin with the P2 tail fiber ${ }^{28}$ supplied guidance. It was hypothesized that the region between two InterPro IPR005068 domains could be a region of interest for fusions. This domain is highly conserved across different tail fibers within Enterobacterales species, however often has little to no protein homology divergent tail fibers. Using an internal Enterobacterales genome database, numerous tail fibers were found containing the IPR005068 domain. Fusions designed with Tfib P1S $_{\text {s }}$ at the second IPR005068 domain were constructed using TFDVs. Many of these fusions resulted in functional Smarticles, with Banana (from E. cloacae strain Ecl16437), Rio (from P. mirabilis strain Pms15785) and Gold (from S. marcescens strain Sms0004) being the most useful for overall Enterobacterales inclusivity (Supplementary Figure S11, Table S2). Amino acid sequences are listed in Supplemental Sequences (Sequence S1-S4, Sequence S9-S10). 


\section{Genomic tail fiber expression constructs}

In order to express chimeric tail fibers from the P1 genomic locus, the non-native tail fiber gene is placed onto the P1 genome in the $E$. coli strain SEG_281 background, which contains a spectinomycin resistance gene in place of the native tail fiber operon. This was accomplished using a $\lambda$-RED recombineering ${ }^{46,47}$ protocol utilizing plasmid pKM208 with the following modifications. The tail fiber gene, tail fiber assembly protein gene, and hygromycin resistance cassette were PCR amplified individually and assembled using the Gibson assembly method. The resulting linear fragment was amplified by PCR with oligonucleotides harboring 5'-homologous sequences specifying the desired $\mathrm{P} 1$ genome integration site. Electrocompetent E. coli SEG_281 cells were transformed with $1 \mu \mathrm{g}$ of the linear DNA fragment. After recovery, cells were plated on hygromycin-selection media. Clones were screened for hygromycin resistance and spectinomycin sensitivity. Candidates were confirmed by PCR and sequence verified. Confirmed clones were subsequently transformed with a pReporter plasmid and selected on zeocin. Ten positive transformants were evaluated for Smarticles production to select the highest titer clone. For each clone, $5 \mathrm{~mL}$ volume lysates were generated and performance was tested in the RLU assay following the protocol below. Each small volume lysate was diluted two-, four, and eightfold in assay media ( $10 \mathrm{~g} / \mathrm{L}$ tryptone, 5 $\mathrm{g} / \mathrm{L}$ yeast extract, $5 \%$ PEG8000, $2.84 \%$ glucose). The RLU assay was performed as described below and light production in three different Smarticles-sensitive strains was determined for the undiluted and diluted lysates. The best lysate, and thereby transformant was determined by the observed light intensity in the luciferase assay. This transformant was subsequently used as production strain for Smarticles lysate generation.

\section{Minimal Inhibitory concentration references}

Vitek2 Card - GN82 AST Card from bioMérieux (Marcy-l'Étoile, France) and testing was performed per the manufacturer's instructions. Reference microbroth testing was performed according to the CLSI M07-A10 document.

\section{Protein similarity and multiple sequence alignments}

Protein sequence identity and similarity of the native tail fibers generated using Matgat BLOSSOM50 ${ }^{48}$. Data on the upper right present the sequence identity and data on the bottom left present the sequence similarity. Multiple Alignment of the native tail fiber amino acid sequences using T-Coffee ${ }^{49}$ (http://tcoffee.crg.cat/apps/tcoffee/do:regular).

\section{Phylogenetic tree}

Tail fiber evolutionary history was inferred using the Neighbor-Joining method ${ }^{50}$. The optimal tree with the sum of branch length $=2.59658478$ is shown. The evolutionary history ${ }^{51}$ was inferred using the Neighbor-Joining method. The optimal tree with the sum of branch length $=2.59658478$ is shown. The percentage of replicate trees in which the associated taxa clustered together in the bootstrap test ${ }^{52}$ (1000 replicates) are shown next to the branches. The evolutionary distances were computed using the $p$ distance method and are in the units of the number of amino acid differences per site. This analysis involved seven amino acid sequences. All ambiguous positions were removed for each sequence pair (pairwise deletion option). There were a total of 1187 positions in the final dataset. Evolutionary analyses were conducted in MEGA X ${ }^{53}$. 


\section{Predicted structural comparison to Mu Tfib and Tfa complex}

Structures for Tfib P1S $_{\text {(GenBank AAQ14006.1), Tfa }}$ (GenBank YP_006501.1), and Tfib Shamrock $_{\text {Sere }}$ predicted using the Protein Homology/analogY Recognition Engine V 2.0 (PHYRE2) ${ }^{54}$ based on the crystal structure of the C-terminal region (residues 138-504) of the bacteriophage Mu tail fiber protein or the

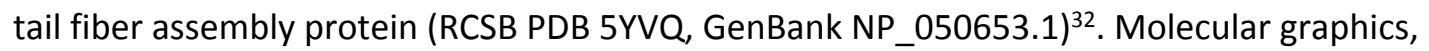
superposition and molecular surface prediction were performed using UCSF Chimera, developed by the Resource for Biocomputing, Visualization, and Informatics at the University of California, San Francisco, with support from NIH P41-GM10331155. The tail fiber proteins of Mu, P1, and Shamrock show nearly identical structures, with notable variations in the binding loops visible at the distal tip (Figure S12).

\section{Supplementary References}

(42) Gibson, D. G., Young, L., Chuang, R.-Y., Venter, J. C., Hutchison, C. A., and Smith, H. O. (2009) Enzymatic assembly of DNA molecules up to several hundred kilobases. Nat. Methods 6, 343-345.

(43) Shanks, R. M. Q., Caiazza, N. C., Hinsa, S. M., Toutain, C. M., and O'Toole, G. A. (2006)

Saccharomyces cerevisiae-based molecular tool kit for manipulation of genes from gram-negative bacteria. Appl. Environ. Microbiol. 72, 5027-5036.

(44) Jakhetia, R., and Verma, N. K. (2015) Identification and Molecular Characterisation of a Novel Mu-

Like Bacteriophage, SfMu, of Shigella flexneri. PloS One 10, e0124053.

(45) Haggård-Ljungquist, E., Halling, C., and Calendar, R. (1992) DNA sequences of the tail fiber genes of bacteriophage P2: evidence for horizontal transfer of tail fiber genes among unrelated bacteriophages. J. Bacteriol. 174, 1462-1477.

(46) Murphy, K. C., and Campellone, K. G. (2003) Lambda Red-mediated recombinogenic engineering of enterohemorrhagic and enteropathogenic E. coli. BMC Mol. Biol. 4, 11.

(47) Thomason, L. C., Sawitzke, J. A., Li, X., Costantino, N., and Court, D. L. (2014) Recombineering: genetic engineering in bacteria using homologous recombination. Curr. Protoc. Mol. Biol. 106, 1.16.1-39. (48) Campanella, J. J., Bitincka, L., and Smalley, J. (2003) MatGAT: an application that generates similarity/identity matrices using protein or DNA sequences. BMC Bioinformatics 4, 29.

(49) Notredame, C., Higgins, D. G., and Heringa, J. (2000) T-Coffee: A novel method for fast and accurate multiple sequence alignment. J. Mol. Biol. 302, 205-217.

(50) Saitou, N., and Nei, M. (1987) The neighbor-joining method: a new method for reconstructing phylogenetic trees. Mol. Biol. Evol. 4, 406-425.

(51) Nei, M., and Kumar, S. (2000) Molecular evolution and phylogenetics. Oxford University Press, Oxford; New York.

(52) Felsenstein, J. (1985) CONFIDENCE LIMITS ON PHYLOGENIES: AN APPROACH USING THE BOOTSTRAP. Evol. Int. J. Org. Evol. 39, 783-791.

(53) Kumar, S., Stecher, G., Li, M., Knyaz, C., and Tamura, K. (2018) MEGA X: Molecular Evolutionary Genetics Analysis across Computing Platforms. Mol. Biol. Evol. 35, 1547-1549.

(54) Kelley, L. A., Mezulis, S., Yates, C. M., Wass, M. N., and Sternberg, M. J. E. (2015) The Phyre2 web portal for protein modeling, prediction and analysis. Nat. Protoc. 10, 845-858.

(55) Pettersen, E. F., Goddard, T. D., Huang, C. C., Couch, G. S., Greenblatt, D. M., Meng, E. C., and Ferrin, T. E. (2004) UCSF Chimera--a visualization system for exploratory research and analysis. J. Comput.

Chem. 25, 1605-1612 


\section{Supplementary Figures}

a

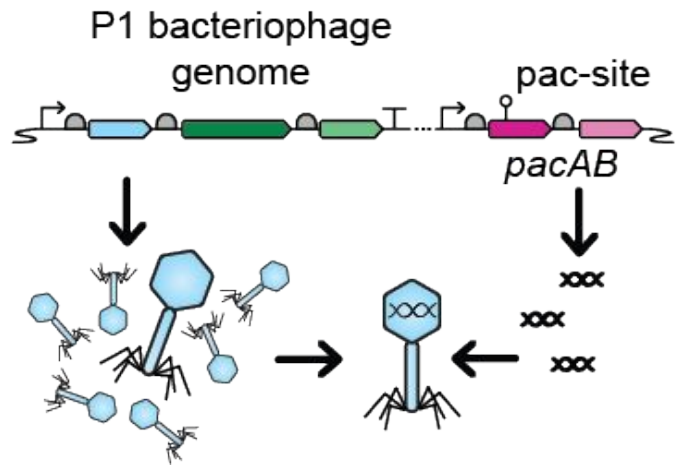

b

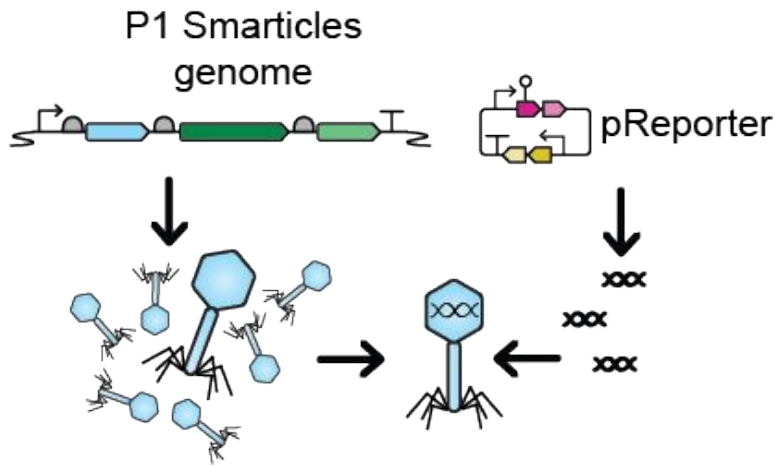

Supplementary Figure S1. Packaging in P1 bacteriophage and P1 based Smarticles a, Structural proteins are produced from the $\mathrm{P} 1$ genome as well as pacase packaging complex proteins PacAB. Packaging recognition site lies within pacA gene and is used to package the $\mathrm{P} 1$ genome into mature bacteriophage particles. $\mathbf{b}$, Smarticles genomes do not contain pacA $B$ genes or packaging site. Pacase packaging complex is supplied in trans on the pReporter plasmid, which also contains packaging site. The pReporter plasmid is packaged into mature Smarticles particles instead of the Smarticles genome. 


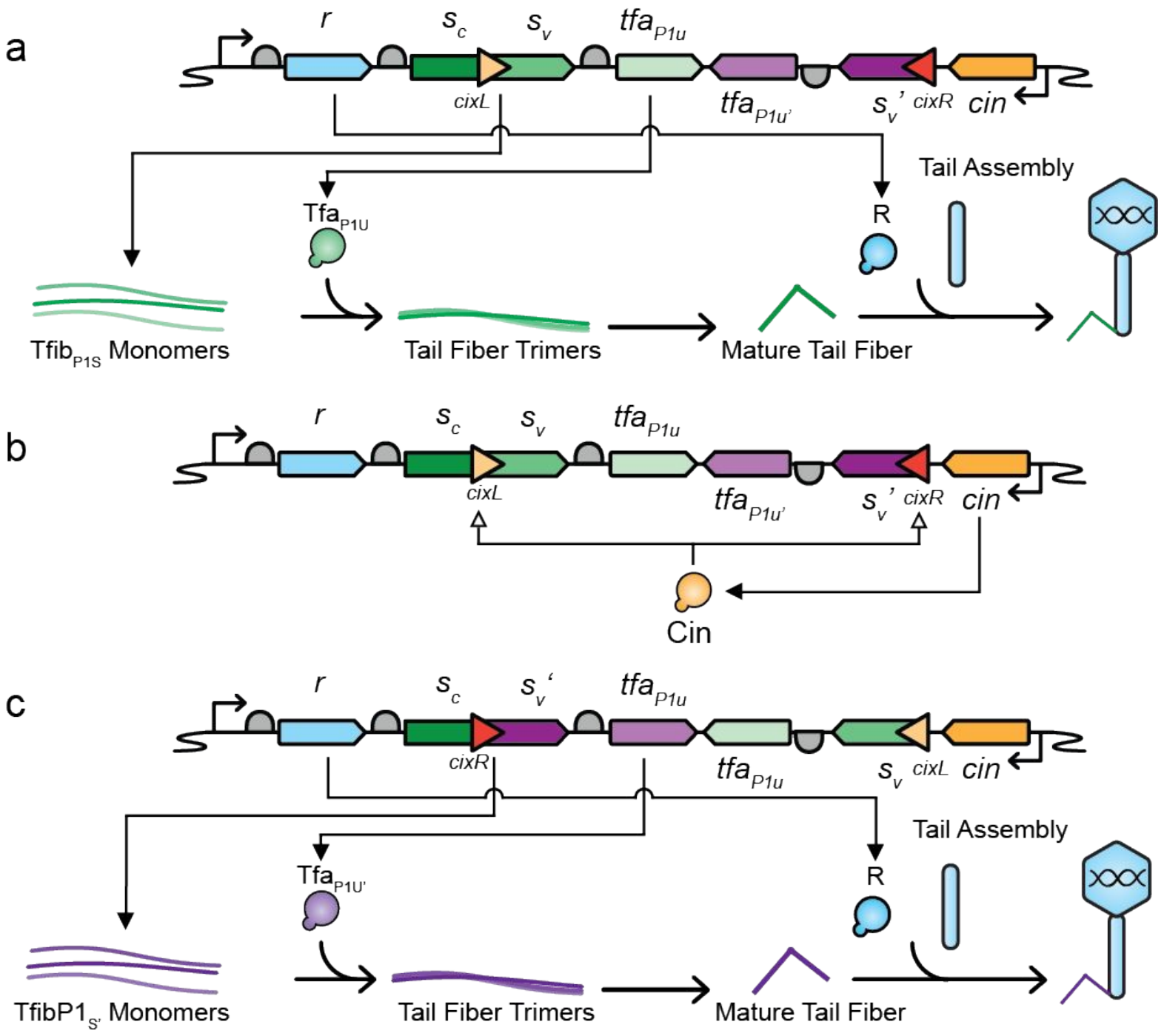

Supplementary Figure S2. Expression of $\mathrm{Tfib}_{\mathrm{P} 1 \mathrm{~S}^{-}}-\mathrm{Tfa}_{\mathrm{p} 1 \mathrm{~S}}$ or $\mathrm{Tfib}_{\mathrm{P} 1 \mathrm{~S}^{\prime}}-\mathrm{Tfa}_{\mathrm{P} 1 \mathrm{~S}^{\prime}}$ variants of tail fibers via Cin-mediated site-specific recombination. a, Tail fiber operon in $t f i b_{P 1 s}$ expression orientation with the main structural gene $t f i b_{P 1 s}$ divided by recombination site cixL to make two halves $s_{c}$ and $s_{v}$. Coded on the opposite strand is the variable portion for expression of Tfib ${ }_{\mathrm{P} 1 s^{\prime}}$ tail fiber, $s_{v}{ }^{\prime}$ and $u^{\prime}$. The $s_{c}$ is notably only in single copy and is required for the variable region expression as the variable regions are not complete open reading frames. b, Upon cin expression, the Cin recombinase acts on the cixL and cixR sites to inverse the DNA strand. c, The result of cin recombination allows for expression of the Tfib ${ }_{\text {P1s }}$ tail fiber variant as the $s_{c}$ and $s_{v}{ }^{\prime}$ regions are downstream with the tail fiber operon promoter. 


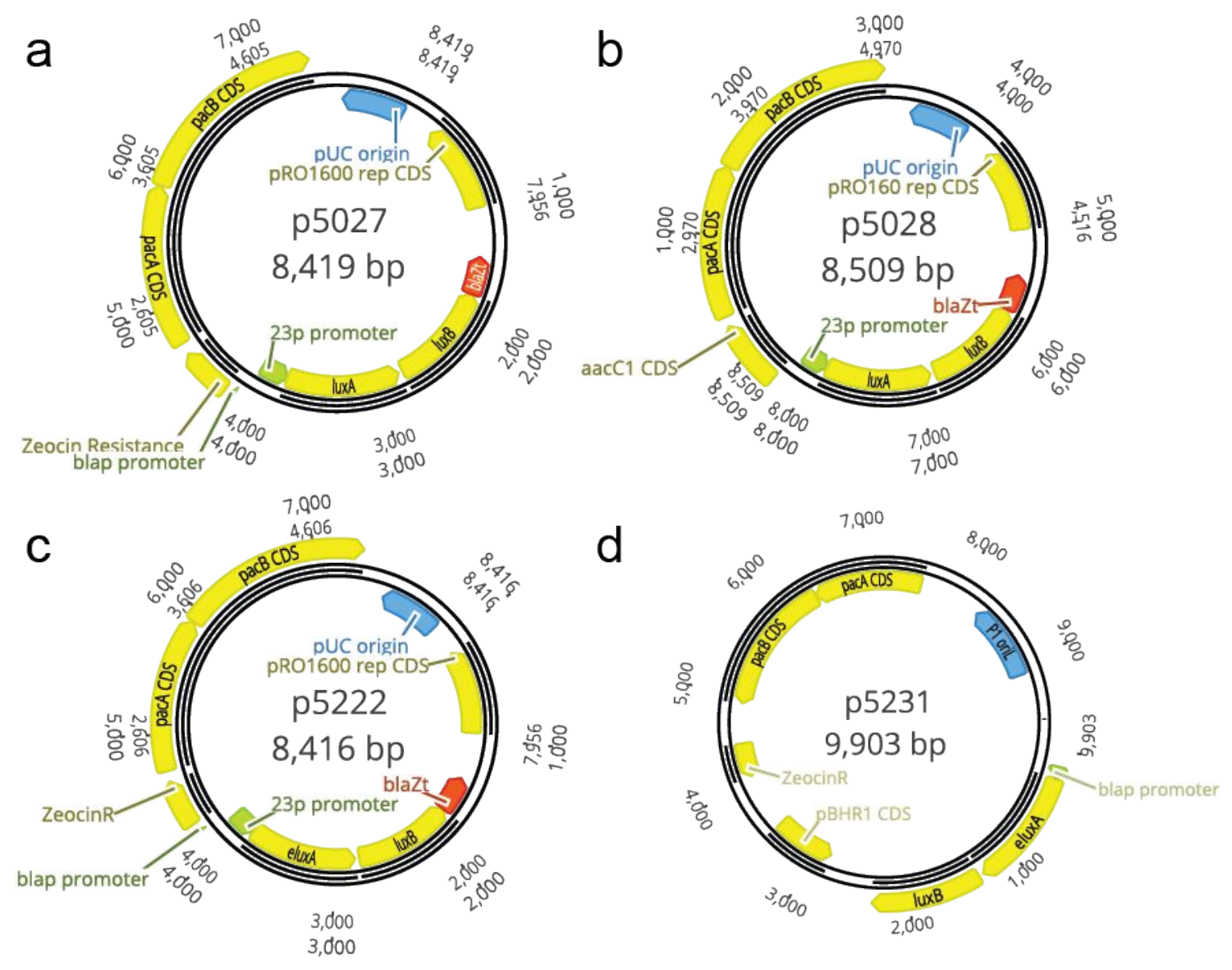

Supplementary Figure S3. Plasmid maps of pReporter variants a, plasmid p5027 containing luxAB, zeocin resistance, and pUC/pRO1600 origins b, plasmid p5028 containing lux $A B$, gentamicin resistance, and pUC/pRO1600 origins c, plasmid p5222 carrying enhanced luxAB, zeocin resistance, and pUC/pRO1600 origins d, plasmid p5231 carrying enhanced luxAB, zeocin resistance, and $\mathrm{pBHR} 1$ origin. 
1. Banana TF

2. Gold TF

3. Jazzberry TF

4. Orchid TF

5. Rio TF

6. Shamrock TF

7. Tangerine TF

8. P1 S TF

9. P1 S'TF

\begin{tabular}{|r|r|r|r|r|r|r|r|r|}
\hline 1 & 2 & 3 & 4 & 5 & 6 & 7 & 9 \\
\hline & 24 & 20.8 & 19.9 & 18 & 20.4 & 24.1 & 20.1 & 21.1 \\
\hline 40.5 & & 16.3 & 19 & 20.2 & 25.1 & 20.7 & 20.6 & 21.3 \\
\hline 31.7 & 26.7 & & 52.3 & 19.2 & 14.8 & 34.2 & 51.7 & 52.3 \\
\hline 33.7 & 28.5 & 62 & & 17.6 & 16.1 & 36.1 & 87.1 & 64.4 \\
\hline 36.6 & 36.1 & 30.1 & 30.3 & & 16.1 & 22.8 & 18.3 & 18 \\
\hline 32.4 & 40.3 & 21.3 & 24 & 27.4 & & 17.6 & 16.8 & 34.6 \\
\hline 40.9 & 36.1 & 42.8 & 44.9 & 40.3 & 28.9 & & 37.7 & 38.1 \\
\hline 35 & 30.5 & 59.9 & 59.8 & 32.5 & 24.8 & 47.4 & & 72.9 \\
\hline 35.1 & 32.5 & 60.2 & 72.5 & 31.7 & 39.5 & 47.4 & 79.8 & \\
\hline
\end{tabular}

Supplementary Figure S4. Protein sequence identity and similarity of the native tail fibers generated using Matgat BLOSSOM50. Data on the upper right present the sequence identity and data on the bottom left present the sequence similarity.

Banana

Gold

Jazzberry

Orchid

Rio

Shamrock

Tangerine

P1 S

P1 $S^{\prime}$

FKYYS ILTNRGKELETQSSATGKPVI IKDFVVGDGNGQAVKPDPAQTKLVREVYRSAI MTAKYRALLTEQGKALLANAAATSQKLEITHMAVGDGGGSPTQPDESQTKLVNEKRRAEL INDVTVVTSVTYPSPESLALVADVQYHEPYLSAALNRKFRGIVDPGFYAGFLPKPGGGMN INDVTVVTSVTYPSP ESLALVADVQYHEPYLSAALNRKFRGIVDPGFYAGFLPKPGGGMN SSQSAITLAFERWKASATEAQQPVI LDEFVLANVPNLDPSKPIDRNEGLPDAKY IMHRQA MFYIDNDSGVTVMPPVSAQRSA A VRWWESEGDGNNVITWPGMDWFNIVQAELLNTLEEAGI MNDVTVVTSVTYPSPESLALVADVQYHEPYLSAALNRKFRGIVDPGFYAGFLPKPGGGMN ESLALVADVQYHEPYLSAALNRKFRGIVDPGFYAGFLPKPGGGMN ESLALVADVQYHEPYLSAALNRKFRGIVDPGFYAGFLPKPGGGMN

Banana

Gold

Jazzberry

Orchid

Rio

Shamrock

Tangerine

P1 S

P1 $S^{\prime}$
61 SALQISPDQANQFIAQLVLPVNVGGFVVREVGLLTDAGELYSVANCAAIEKPENGVSVNL 61 NSLQIDTGNSNQVIÄEQVIPEDVGGWWIRELGLYDKNGVLVAIANTPDTYKPQLTEGAGR 61 LLITSVDGDKTAGAASVDIGEFYQVTIQHRKDISLALSAGKKYAIVLKGRYLLGEDTYQV 61 LLITSVDGDKTAGAASVDIGEFYQVTIQHRKDISLALSAGKKYAIVLKGRYLLGEDTYQV 61 VNKTGVVNQNAVAYSVTIGAEIGDFDFNW I GLLNKQS GTVAMIVHAPTQRKIKTQAGQQG 61 QPDKTKLNQLALSIKAIMSNNALLIKNNLSEIKTAGASAQRTARENLDIYDASLNKKGLV 61 LLITSVDGDKTAGAASVDIGEFYQVTIQHRKDISLALNAGKKYAIVLKGRYLLGEDTYQV 61 LLITSVDGDKTAGAASVDIGEFYQVTIQQRKDISLALSAGKKYAIVLKGRYLLGEDTYQV 61 LLITSVDGDKTAGAASVDIGEFYQVTIQQRKDISLALSAGKKYAIVLKGRYLLGEDTYQV
Banana Gold

Jazzberry Orchid

Rio

Shamrock

Tangerine
121 QYRLAVISETAAVLKVATGDGIFLRLDRNLGEIAENGPSAQKSAREAIDVVDATTSRKGL 121 TQVVRMVLLVKGDANAA IVADKTALLVSRDTLSAAIAEHARSRNHPDATLLAKGFTQLSN 121 NTASHIHAAEFVARTYTDSYQLGDGELLVCTVNIPAGVSA ITQEMIDTSERINRTIGIDI 121 NTASHIHAAEFIARTYTDSYQLGDGELLVCTVNI PAGVSAITQEMIDTSERINRTIGIDI 121 NVLTRSFLLEYLGASKETAITTPAEMWQIDFTARLSGIDEMQRLINRDKYGVASFFNNGF 121 QLTSATDSPSETLAATAKAVKIAMDNASARLAKDRNGADI PNKPLE IQNVGLQETVNQAS 121 NTASHIHAAEFVARTYTDSYQLGDGELLVCTVNIPAGVSTITQEMIDTSERINRTIGIDI 

P1 S
121
P1 $S^{\prime}$
121
NTASHIHAAEFVARTYTDSYQLGDGELLVCTVNIPASVST
VSAITQEMIDTSERINRSIGIDI

$S^{\prime}$

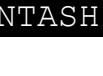
Banana $\quad 181$ VQLSNATDTTSEMAATPKAVKAAYDLADGKYTÄLDATTARKGIVQLSSATDSTSEALAA Gold $\quad 181$ DSNSGSETLAATPKAVKAVNDASLKIAANLKDLPNKSVARGNLELGTAATRNVGAQKTNL Jazzberry 181 SDSVTSSRSDVAASSLAVKKAYDLAKSKYTAQDASTTQKGLVQLSSETNSDSETMAATPK Orchid 181 SDSVTSTRSDVAASSLAVKKAYDLAKSKYTAQDASTTQKGLVQLSSATNSDSETMAATPK RiO $\quad 181$ LVAKAGNQFYITKGLGYIEGLRAELAENKNITVTNQATKVWVDVSYQGNITSDWGVHIAI Shamrock 181 GALQKNQNGADI PGKDTFTKNIGACRAYSAWVDIGGDSQVWTTAQFISWLESQGAFNHPY Tangerine 181 SDSVTSTRSDVAASSLAVKKAYDLAKSKYTAQDASTTQKGLVQLSSATNSTSEVLAATPK P1 S 181 SDSVTSTRSDVAASSLAVKKAYDLAKSKYTAQDASTTQKGLVQLSSATNSDSETMAATPK
P1 $\mathrm{S}^{\prime} \quad 181$ SDSVTSTRSDVAASSLAVKKAYDLAKSKYTAQDASTTQKGLVQLSSATNSDSETMAATPK

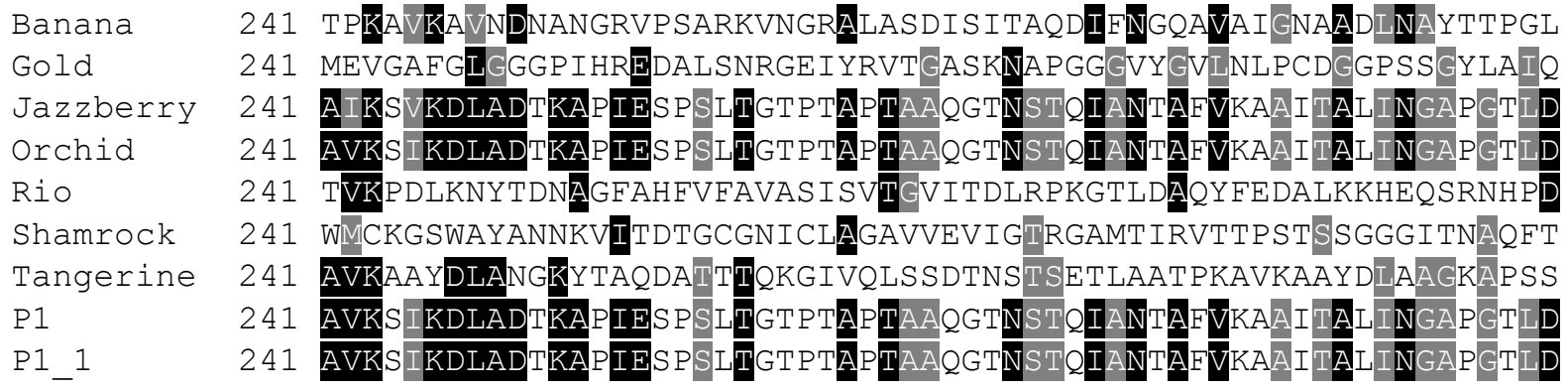
Banana $\quad 301$ YYQPANAAQETGRNYPEANAGSLEVYKHAGITQIYRIYNSSRSYIRTLYSGTWSAWVKQY Gold $\quad 301$ PNGSSYITSTTPDKPLNWYRIYTTGEKPTATDVDAYSKAEADGKFVKOSGDTITGALTV Jazzberry 301 TLKEIAAAINNDPNFSTTINNALALKAPLASPALTGIPTAPTAAOGTNNTQIATTAYVRA Orchid $\quad 301$ TLKEIAAAINND PNYSTTINNALALKAPLASPALTGVPTAAPAACGTNNTQIATTAYVRA RiO $\quad 301$ ATTTVKGFTKLNNQVGDSENTALTPYGAKKELENYQPKGNYQPAGNYVTTATFNLEINKK

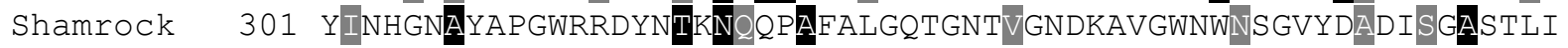 Tangerine 301 HTHPWNQITGVPTASLTAKGITQLSSATNSTSEVLAATPKAVKAAYDLANGKYTAQDATT P1 S 301 TLKEIAAAINNDPNYSTTINNALALKALAASPALTGVPTAPTAACGTNNTEIATTAYVRA P1 S' $\quad 301$ TLKEIAAAINNDPNYTTINNALALKAPLASPALTGVPTAPTAAQGTNNTQ IATTAYVRA 
Banana

Gold

Jazzberry

Orchid

Rio

Shamrock

Tangerine

P1 S

P1 $S^{\prime}$

Banana

Gold

Jazzberry

Orchid

Rio

Shamrock

Tangerine

P1 S

P1 $S^{\prime}$

Banana

Gold

Jazzberry

Orchid

Rio

Shamrock

Tangerine

P1 S

P1 $S^{\prime}$

Banana

Gold

Jazzberry

Orchid

Rio

Shamrock

Tangerine

P1 S

P1 $S^{\prime}$

Banana

Gold

Jazzberry

Orchid

Rio

Shamrock

Tangerine

P1 S

P1 $S^{\prime}$
361 DAANKPSPADINAVNKGGDTMTGGLKIRAADALRIYDAAYGMIFRRSENNFYLIPTAKRDQ 361 NGAIESKSGLTTPSLAVNGSVTIAGALTTKAGVELFGTTPYLDFHYGNSNGDFDVRLIND 361 AISALVGSPEALDLNELAAALGNDPNFATTMTNALAGKQPLDATLTALAGLATGANKL 361 AISALVGSPEALDTNELAAALGNDPNFATTMTNALAGKQPLDATLTALÄGATGANKL 361 IDKA ALSSQQLGNDVNKVPSLDLVTKELGNKQPKGDYALKTWVDNIYYKRDDSPSFNEISA 361 LHFNKNTGSCPAVQFRVNYKNGGIFYRSARDGYGFEAGWSEFYTTTRKPSARDVGAYTQA 361 AQKGIVQLSSATNSTSEVLAATPKAVKAAYDLANGKYTAQDATTTQKGIVQLSSDTNSTS 361 A I ALVGSSPEALTLYELAAALGNDPNEATTMTNALAGKQPLDATLTALAGLATGANKL

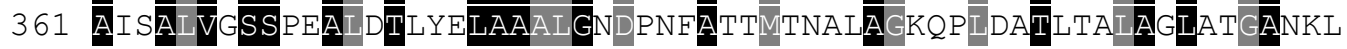

421 GEDGGISGIRPLYIDLTNGRVTLGNGAVVNGGLGLGVVSGLGGNSIALGDNDTGFKQNGD 421 NKGTLAFHGNEYYVNGKLSATGDAWIGGKAN INGMAAFYSSDFITKQGNLTHPDGNRQTN 421 PYFTGTDTVSQTDLTSVGRDILAKTSVIAVIQYLGLRELGTSGEKIPLLSTANTWSARQT 421 PYFTGTDTVSQTDITSVGRDILAKTSVIAVIQYLGLRELGTS GEKIPLISTANTWSSRQT 421 TAIRVNSVNVALKGDCYTKSESDNKYSPKLPFVYSSGEESKI ISPNNKILIFVNDNGTTG 421 ECNSRFITGIRLGGLSSVRTWNGPGWSDRSGYVVTGSVNSNRDEIIDTTQARPVQYCING 421 ETLAATPKAVKAAYDLAAGKAPSSHTHPWNQITVVPTASLTAKGITQLSSATNSTSEVLA 421 PYFTGTDTVSQTDLTSVGRDILAKTSILAVIQYLGLRELGTSGEKIPLLSTANTWSARQT 421 PYFTGTDTVSQTDLTSVGRDILAKTSILAVIQYLGLRELGTSGEKIPLISTANTWSARQ T

481 GVLDVYANSKQVMRELNSGITSYMLFNMNAGASVSSTLTEKNGSGITSEKTGANPRNGRI 481 GMRLQGQGNLLVDLYHYEKVGSHHEFGIHVANGGADGWFSFRNNGELRANGTLFAAGAAY 481 FNGGITGALTGNADTATKLKTARKINNVSFDGSADITLTPENLGVTSLTFEKNNGEMPID 481 FNGGITGALAGNADTATKLKTARSIGGVAFDGSANINLPGVNTTGNQNTTGNAATATKLA 481 GYDRDTOATVWGFDKRGFMTRGTVHFDRMSGVYSQSQVDAKLKECLNLKTSSKQFIASDI

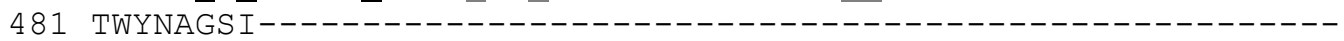
481 ATPKAVKAAYDLANGKYTAQDATTAQKGIVQLSSATNSTSEVLAÄTPKAVKAAYDLANGK 481 FNGGITGALTGNADTATKLKTARNINGVRFDGSGDININTLVSRGRVTALEANAQGTSGI 481 FNGGITGALTGNADTATKLKTARNINGVRFDGSGDININTLVSRGRVTALEANAQGTSGI

541 YWGGDASRGNRIEFADDAGWKAYIERHPSNGVQLVVNGRINGS IVYSSGEVLAGGGSARF 541 QTDGNINGGIWGGYLSNYLNHNFVRDVRLGNVES IATWRGPGYSDSAGYVLTGAANNNVD 541 ADLNTFGPVEAYLGVWSKATSTNATLEKNFPEDNAVGVLEVFAAGNFAGTQRFTTRDGNV 541 TARNINGVKFDGSVDISIPTITSRGRVTALTGTTQGAATGLQMYEAYNNGYPSAYGNVLH 541 YAPTFITGSQYGERSYLEHKSDELTITHISPKSGATNITCRNKSGTLALLGDLEDVNNIP

541 QAADATLTALAALATAADKLPYFTGVDRAALTALTSVGRAILGKTSIQSVLDYLGLGEGS 541 QLYEAYNNGYPSPYGNVLHLKGATAAGEGELFIGWSGTS GAHAPVHIRSRRDTDSANWSE 541 QLYEAYNNGYPSPYGNVLHLKGATAAGEGELFIGWSGTSGAHAPVH IRSRRDTDSANWWSE

601 AADGNIFGSKWGNQWLDAYLKNTYQPKGNYTPAGQAYTKVESDGRFQPKGSYTPAGQAYT 601 EYIDVIFRRPLQKHIGGNWVTVWSV------------------------------601 YIRRLANKWNGSDGPWGIWRHTQSATRPLSTTIDLNTLGAAEHLGLWRNSSSAIASYERN 601 LKGATAVGEGELFIGWSGTSGAHAPVHVRSRRDTDTASWSEWAQVYTSKDS I PGVNTTGN 601 VGSPIPWSLATAPSGYLICNGQTFNKSTYPKLLAVAYPSGKLPDLRGEFIRGLDSGRGIDA

601 ALPVGVPVPWPSATPPTGWLKCNGAAFSSEKYPNLAKVYPTLKLPDLRGEFIRGWDDSRG 601 WAQVYTSKDSIPGVNAKGDQDTSGNAATATKLQTACT INGVSFDGSKNIELTAEDLNLQE 601 WAQVYTSKDSIPGVNAKGDQDTSGNAATATKLQTACTINGVSFDGSKNIELTAEDLNLQE 
Banana

Gold

Jazzberry

Orchid

Rio

Shamrock

Tangerine

P1 S

P1 $S^{\prime}$

Banana

Gold

Jazzberry

Orchid

Rio

Shamrock

Tangerine

P1 S

P1 $S^{\prime}$

Banana

Gold

Jazzberry

Orchid

Rio

Shamrock

Tangerine

P1 S

P1 S'

Banana

Gold

Jazzberry

Orchid

Rio

Shamrock

Tangerine

P1 S

P1 $S^{\prime}$

Banana

Gold

Jazzberry

Orchid

Rio

Shamrock

Tangerine

661 KAESDARYNLKNTATKSANAMTHKDASTGVMEVVMSNINVPNKTNVNVTFPAAFPNACVG

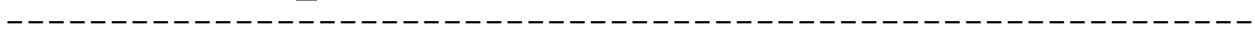

661 YPEEGGFAQGVLEILEGGNYGRTQRYTTRRGNMYVRCLAASWDASNPQWEPWLKVGHQSE 661 QNTTGNAASATKLQTARTIGGVSFNGTANIDLPGVNKTGNQNTTGNAATATKLQTARTIN 661 GRSVLSVQKGNSLLSSNIFGGLSSSESNKWHKAINYIGITGTDNDGGYGVHQQIEGANGN 661 IDTGRSLLSGQTATFIRTALQDYYGVDLTTNVKVGIAYATADSVITVGNPANPKAGDNSD 661 TVNKADNAVQKTGDTLSGGLTFENDS I LAWIRNTDWAKIGFKNDADSDTDSYMWFETGDN 661 TVNKADNAVQKTGDTLSGGLTFENDS ILAWIRNTDWAKIGFKNDADSDTDSYMWFETGDN

721 VVITYNGAGHGSGDDSAIYVPSYSRTGCTLYAHNADGKFMLIAKGY--------------

721 SRYYEGDLNVLTDPGIYSVTGKATNGPMLDTVGATLLGILEVIRREDGVSVWTRYTTTGK 721 GVSFDGSKNIELTAEDLNLQEFINKANNAVQRSGDTLSGGLTFKNDSILAWIRNTDWAKI

721 ETRPRNIAFLYIVRAA---------------------------------------

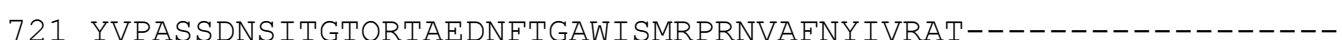

721 GNEYFKWRSKQSTTTKDLMNLKWDALYVLVNAIVNGEVISKSANGLRIAYGNYGFFIRND

721 GNEYFKWRSKQSTTTKDLMNLKWDALYVLVNAIVNGEVISKSANGLRIAYGNYGFFIRND

781 SETTQGRTFERVYAGSKWTEWREVYNSFSLPLNLGIGGAVAKLSSLDWQTYDFVPGSLIT

781 GFKNDADGDTDSYMWEETGDNGNEYFKWRSKQSTTTKDLMNLKWDALYVLVNAIVNGEVI

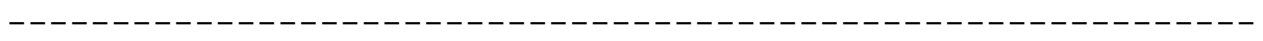

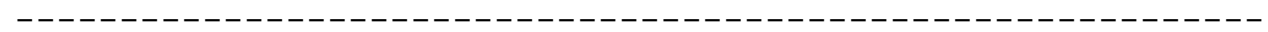

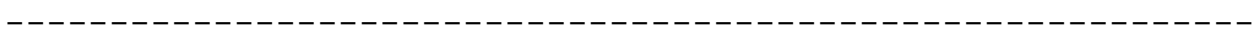

781 GSNTYFMLTNSGDNMGTYNGLRPLWINNATGAVSMGRGLNVSGETLSDRFAINSSNGMWI

781 GSNTYFMLTNSGDNMGTYNGLRPLWINNATGAVSMGRGLNVSGETLSDRFAINSSNGMWI

841 VRLDNMTNIPDGMDWGVIDGNLINIAVGPSDDSGTGRSMHVWRSTVSKANYRFFMVRISG 841 SKSANGLRIAYGNYGFFIRNDGSNTYFMLTNSGDNMGTYNGLRPLWINNATGAVSMGRGL

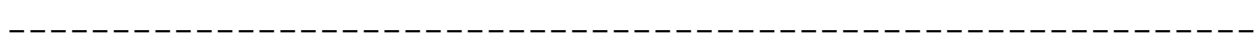

841 QMRDNNAIFGKNIVNTDSTQALLRQNHADRKFMIGGLGNKQFGIYMINNSRTANGTDGQA 841 QMRDNNAIFGKNIVNTDSTQALLRQNHADRKFMIGGLGNKQFGIYMINNSRTANGTDGQA

901 NPGSRTITARRVPIIDEAQTWGAKQTFSAGLSGELSGNAATATKLKTARKINNVSFDGSG

901 NVSGETLSDRFAINSSTGMWINMRDQNVIMGRNAVSTDGAQALLRQDHADRKFMIGGLGN

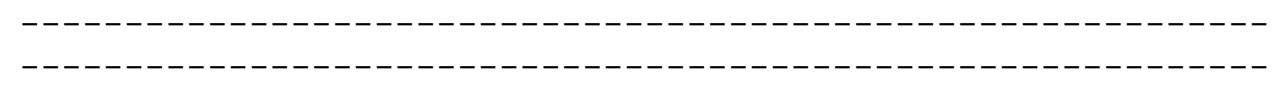


P1 S 901 YMDNNGNWLCGAQIIPGNYGNFDSRYVRDVRLGTRVVQLMARGGRYERAGHALTGLRIIG

P1 S' 901 YMDNNGNWLCGAQIIPGNYGNFDSRYVRDVRLGTRVVQLMARGGRYERAGHALTGLRIIG

Banana

Gold

Jazzberry

Orchid

Rio

Shamrock

Tangerine

P1 S

P1 $S^{\prime}$

Banana

Gold

Jazzberry

Orchid

Rio

Shamrock

Tangerine

P1 S

P1 $S^{\prime}$

Banana

Gold

Jazzberry 1081

Orchid

Rio

Shamrock

Tangerine

P1 S

P1 $S^{\prime}$

Banana

Gold

Jazzberry 1141

Orchid

Rio

Shamrock

Tangerine

P1 S

P1 $S^{\prime}$
961 DIEVLPVGVPLPWPSDTVPSGYALMQGQTFDKSAYPKLAAAYPSGVIPDMRGWTIKGKPA

961 KQFGIYMINNSRTANGTDGQAYMDNNGNWLCGAQI I PGNYGNFDSRYVKDVRLGTRVVQL

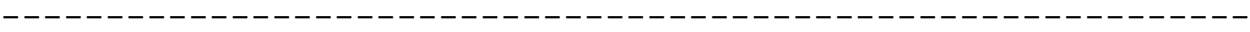

1021 SGRDVISTEODGIKSHTHSASASNTDIGTKTTSSFDYGTKSTNNTGAHTHNVSGTANSAG

1021 MARGGRYEKAGHAITGLRI I GEVDGDDEA I FRPIQKY INGTWYNVAQV------------

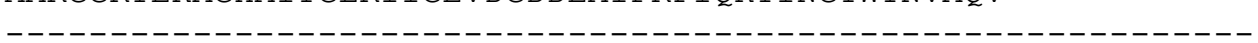
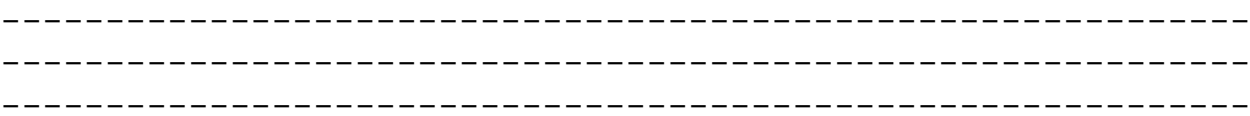

Supplementary Figure S5. Multiple Alignment of the native tail fiber amino acid sequences using T-Coffee. 


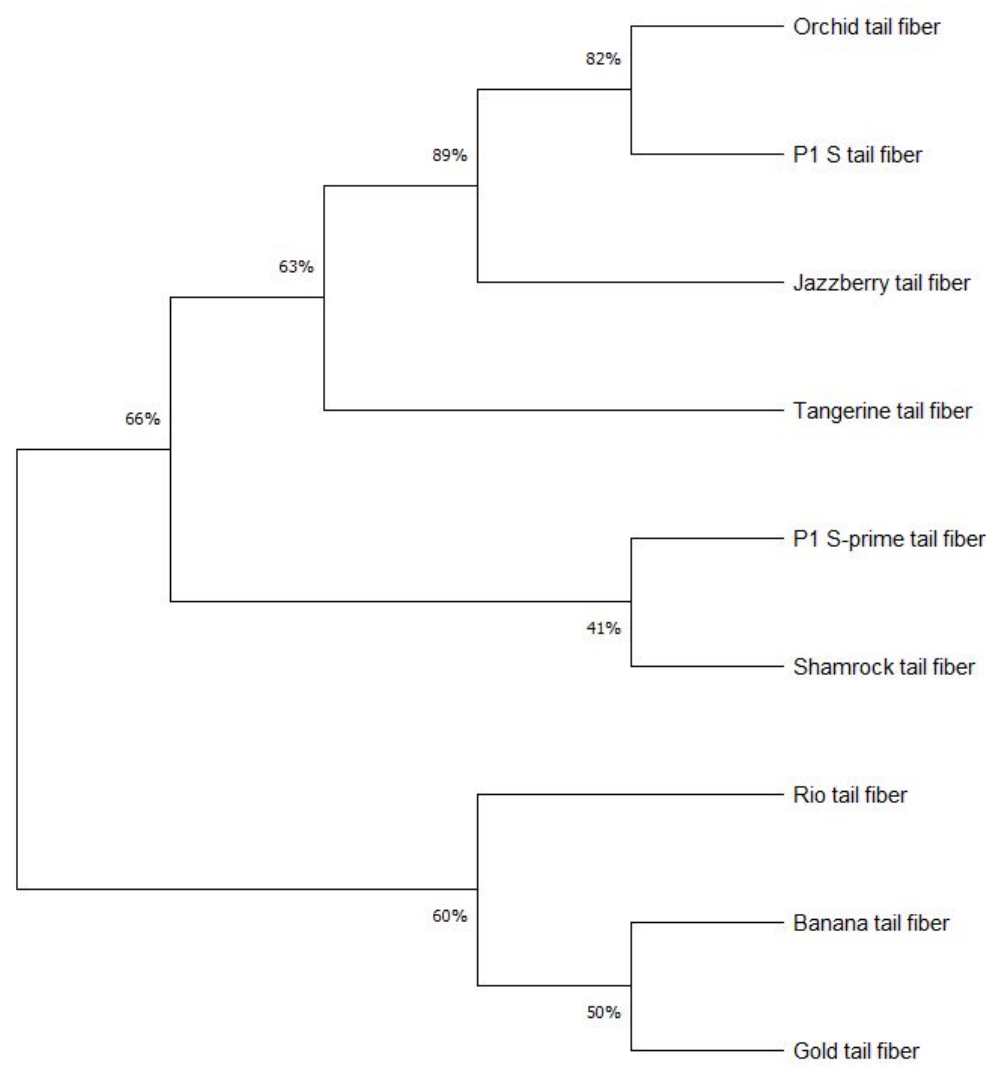

Supplementary Figure S6. Phylogenetic tree of the native tail fiber proteins inferred using the Neighbor-Joining method implemented in MEGAX. The percentage of replicate trees in which the associated tail fiber clustered together in the bootstrap test $(1,000$ replicates) are shown next to the branch. 

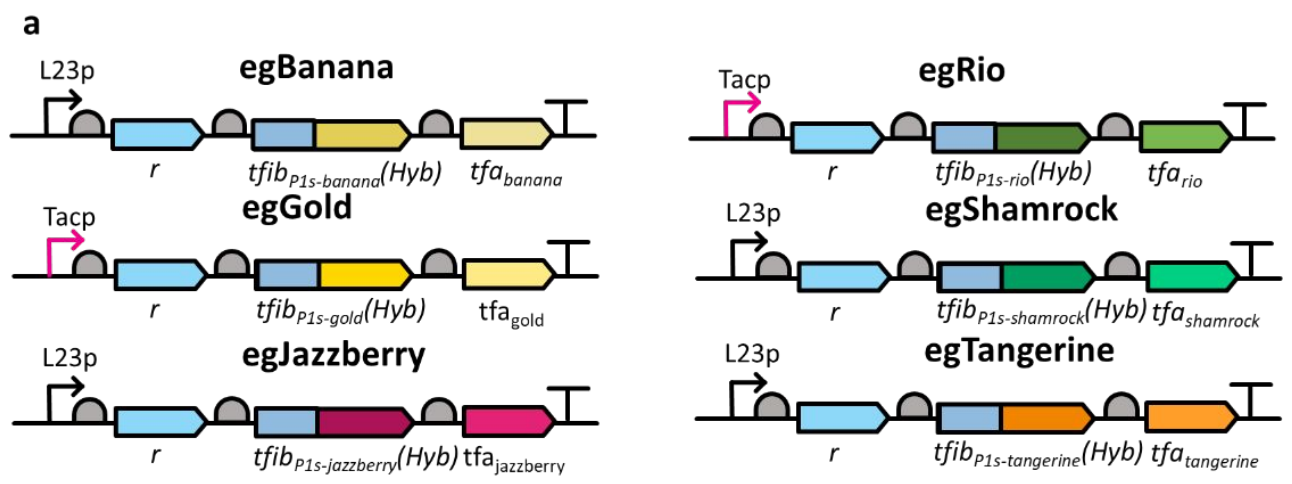

b

egShamJazz

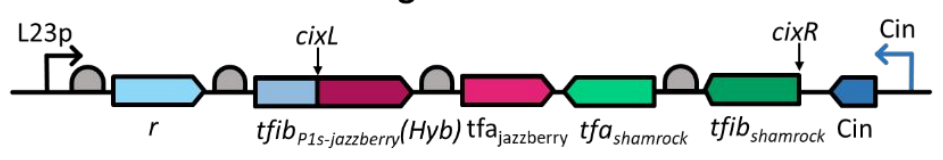

C

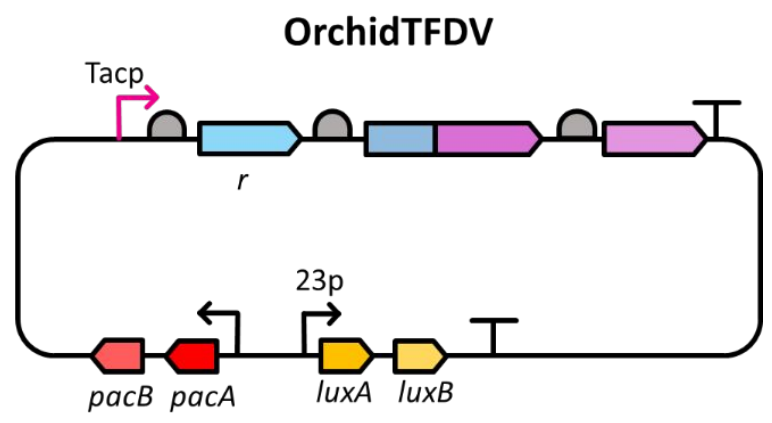

Supplementary Figure S7. a, Schematic representation of genomic expressed egBanana, egGold, egJazzberry, egRio, egShamrock, egTangerine. Native P1 Tfib-Tfa operon promoter L23p or Tacp IPTG-inducible promoter used for expression. b, egShamJazz contains both Shamrock and Jazzberry genes that are expressed via Cin-mediated recombination of the operon. $\mathbf{c}$, Orchid TFDV is not expressed off of the P1 chassis genome, but from a TFDV. 

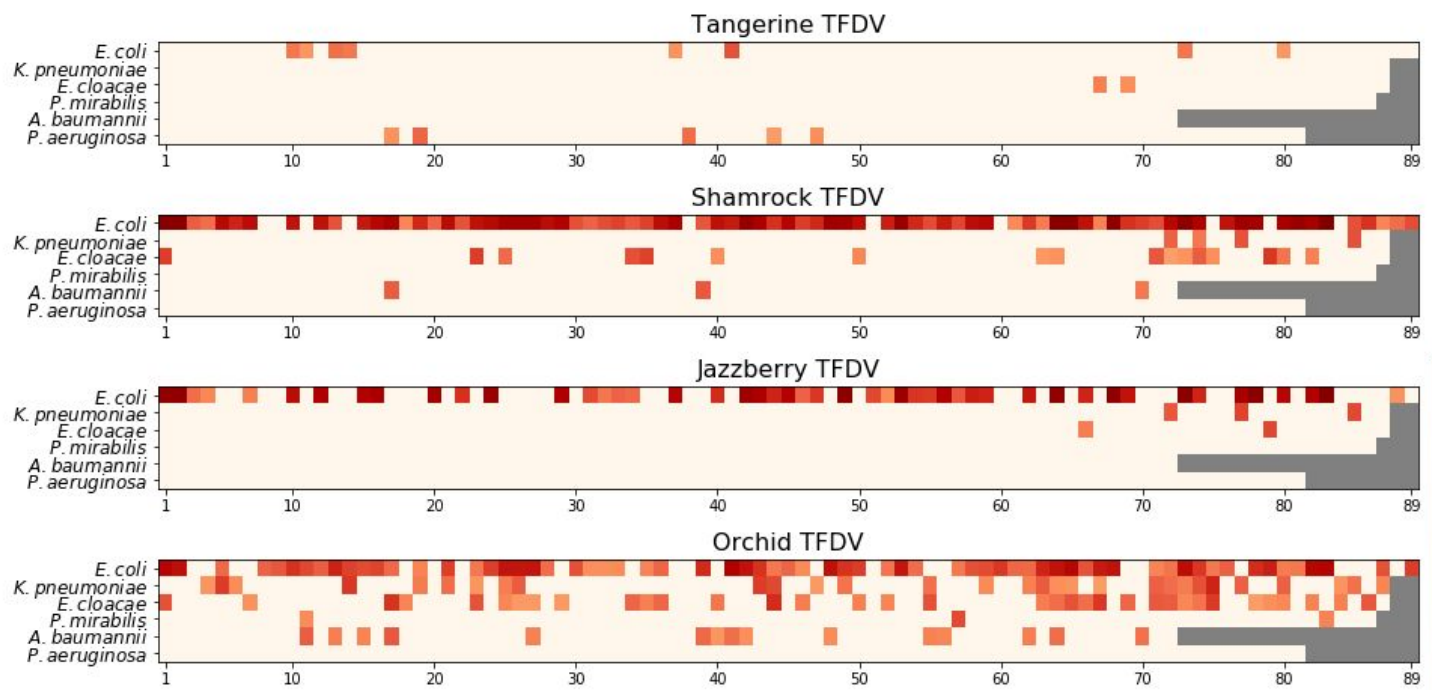

Banana TFDV

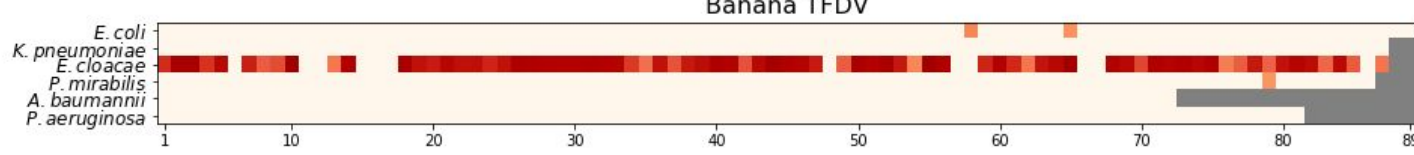
Gold TFDV

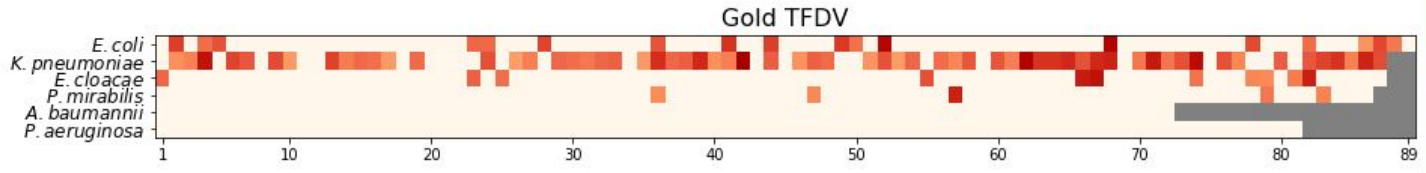
Rio TFDV

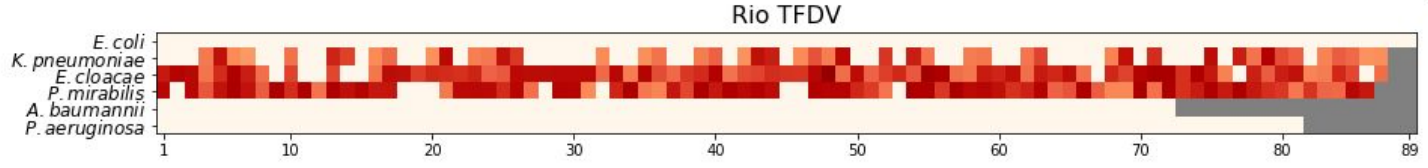
P1 Wild Type Tail Fibers

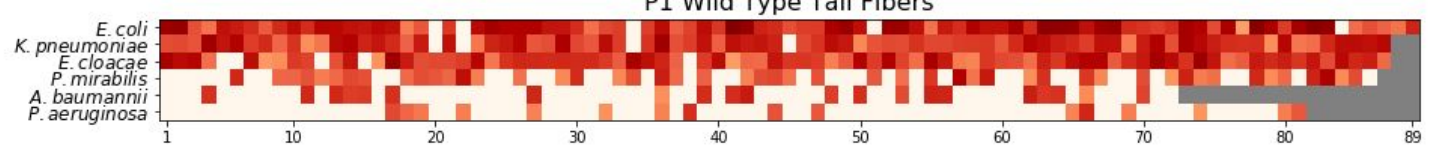

Strain Number

Supplementary Figure S8. Reactivity of TFDV expressed tail fiber on Smarticles chassis. Tail fibers have varying degrees of reactivity on the six panels of Gramnegative bacteria. Tail fibers complement each other to have greater inclusivity on a certain panel. Of note is Gold TFDV and Rio TFDV, which cumulatively have high reactivity of $K$. pneumoniae. Luminescence (RLU) range, color bar. 

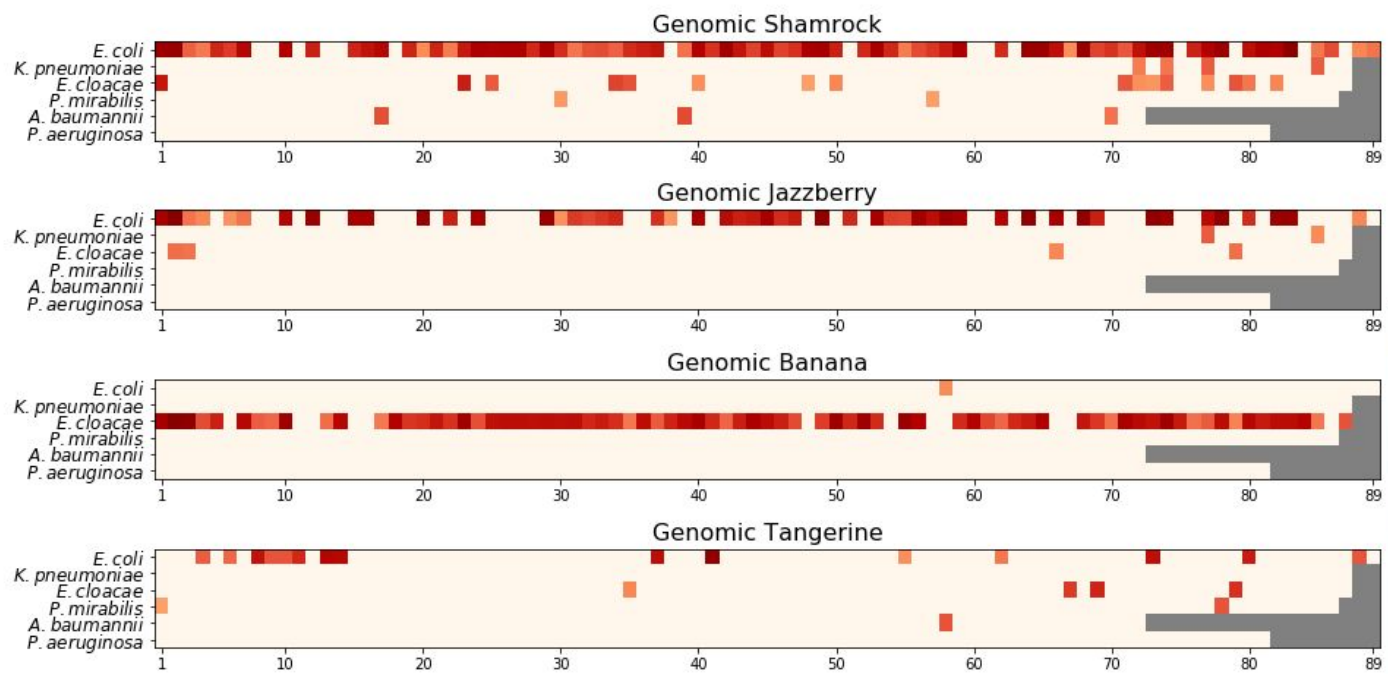

Genomic Rio
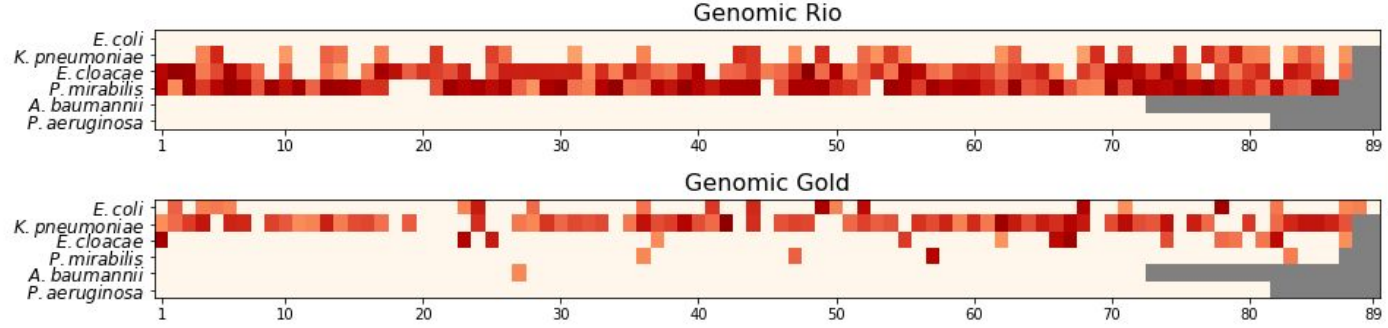

P1 Wild Type Tail Fibers

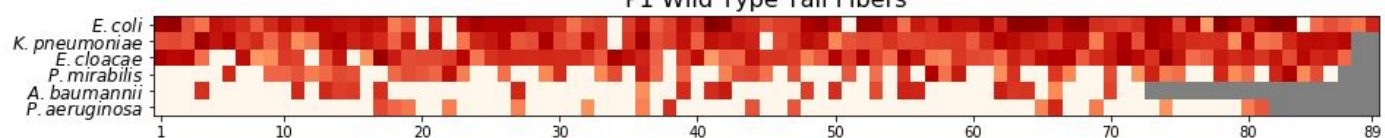

Strain Number

Supplementary Figure S9. Reactivity of tail fiber expressed on Smarticles genomic tail fiber locus. Overall reactivity for each genomic expressed tail fiber is similar to TFDV expressed tail fiber Smarticles. Luminescence (RLU) range, color bar. 


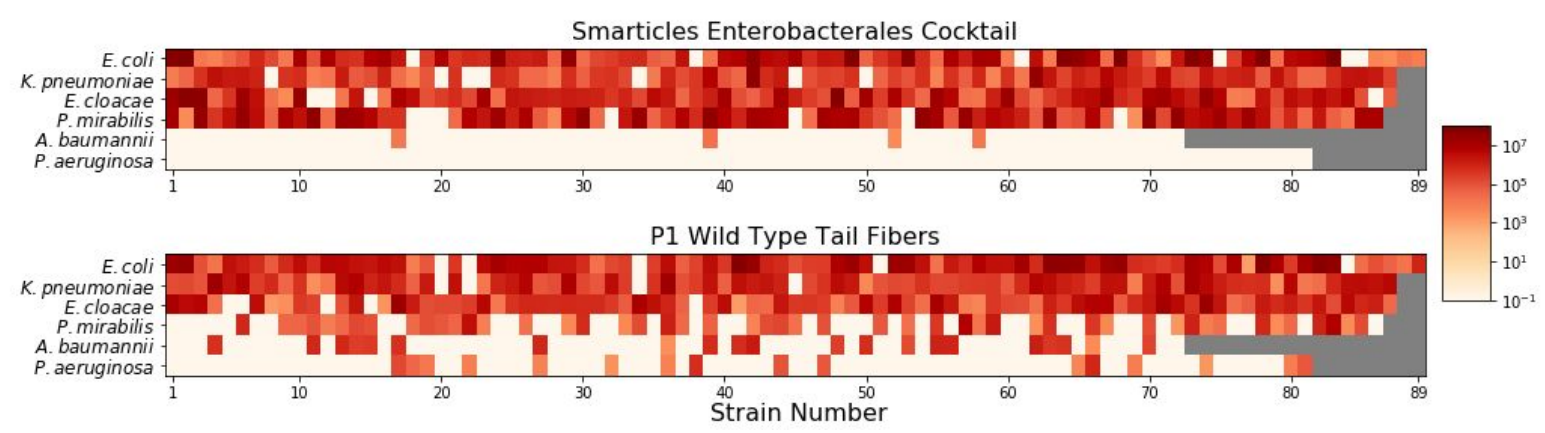

Supplementary Figure S10. Reactivity of Engineered Smarticles cocktail compared to the Smarticles utilizing the wild type $S$ and $S^{\prime}$ tail fibers of $P 1$. Engineered Smarticles cocktail has high reactivity with Enterobacterales species. Little to no reactivity to $A$. baumanii and $P$. aeruginosa. Luminescence (RLU) range, color bar.

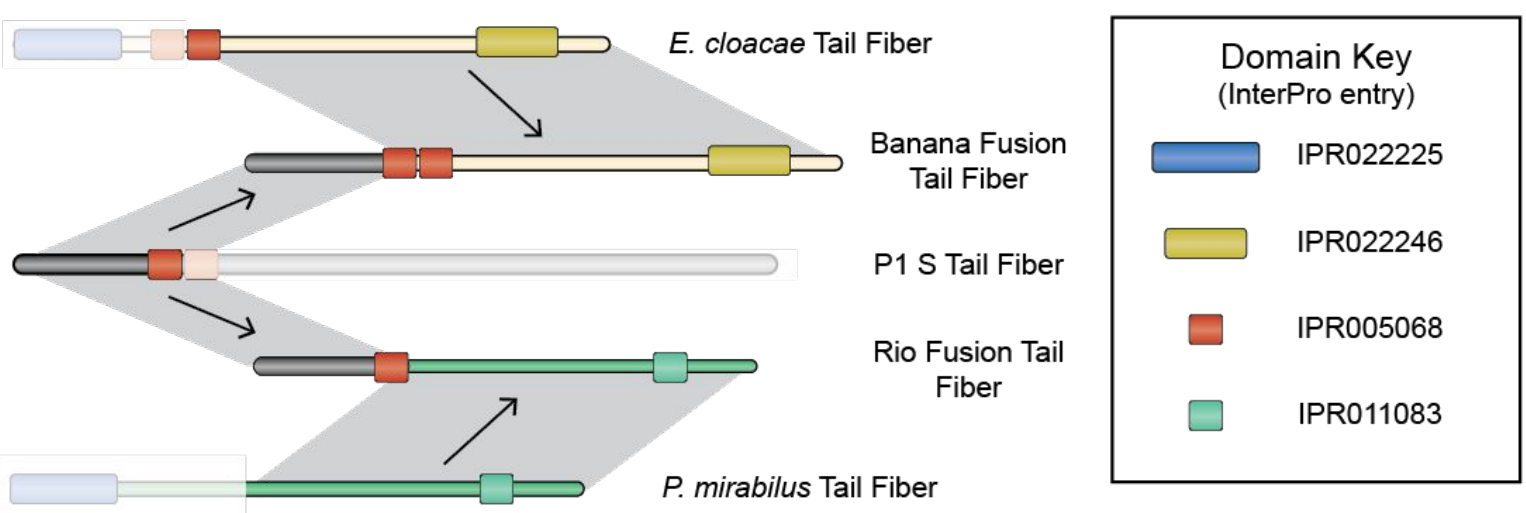

Supplementary Figure S11. Domain architectures of native tail fibers found in $E$. cloacae and $P$. mirabilis phage genomes along with wild-type P1 S tail fiber protein. Fusions of tail fibers used on Smarticles chassis shown for Banana ( $E$. cloacae) and Rio (P. mirabilis). Domains listed by their InterPro entry numbers (https://www.ebi.ac.uk/interpro/). 
a

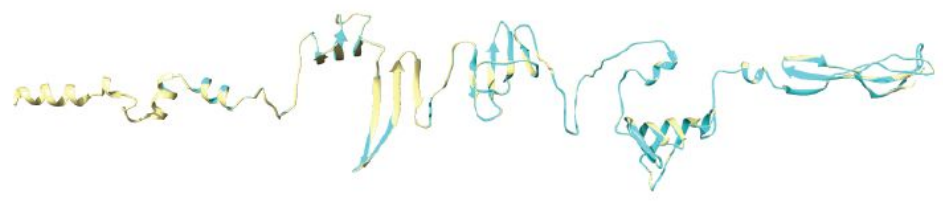

b

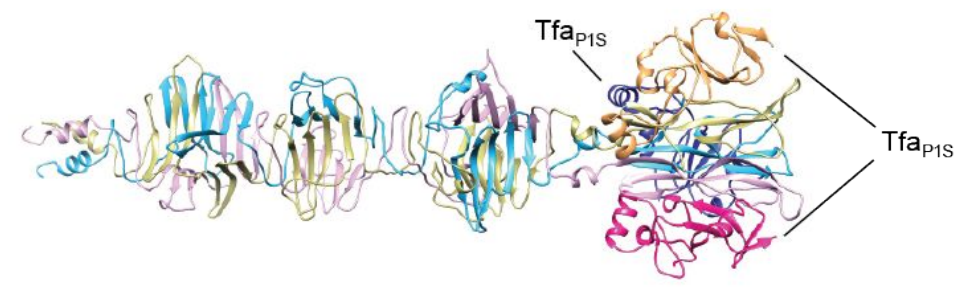

C

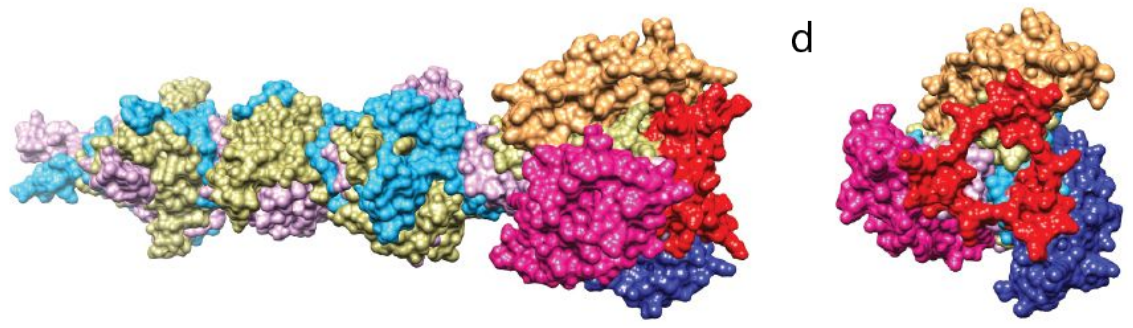

e

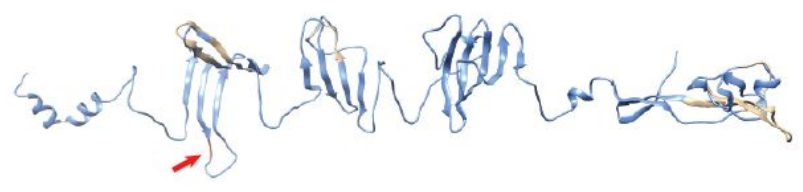

Supplementary Figure S12. Structure comparison with known Tfib $_{\text {MuS }}$ and predicted tail fiber structures. a, Predicted C-terminal ribbon structure of $\mathrm{Tfib}_{\mathrm{P} 1 \mathrm{~S}}$ monomer in beige superimposed on to the known $\mathrm{Tfib}_{\mathrm{Mu}}$ ribbon structure in blue (PDB 5YVQ). A high amount of similarity can be seen between the two structures, with variability in the distal end loops. The loops are predicted by North et al. to be involved in cell surface binding. b, Predicted trimeric ribbon structure of $\mathrm{Tfib}_{\mathrm{P} 1 \mathrm{~S}}$ with $\mathrm{Tfa}_{\mathrm{P} 1 \mathrm{~s}}$. Tfib $\mathrm{P}_{\mathrm{P} 1 \mathrm{~S}}$ trimer is shown with lighter colors of blue, beige and lavender. $\mathrm{Tfa}_{\mathrm{P} 1 \mathrm{~S}}$ is shown in orange, magenta and purple. c, Surface model of Tfib $\mathrm{P}_{\mathrm{P} 1 \mathrm{~S}} \mathrm{Tfa}_{\mathrm{P} 1 \mathrm{~S}}$ structure with $\mathrm{Tfib}_{\mathrm{P} 1 \mathrm{~s}}$ variable loops highlighted in red (residues 937-944,958966). d, Surface model of $\mathrm{Tfib}_{\mathrm{P} 1 \mathrm{~S}} \mathrm{Tfa}_{\mathrm{p} 1 \mathrm{~S}}$ structure from the distal end. e, Predicted ribbon structure of $\mathrm{Tfib}_{\mathrm{P} 1 \mathrm{~S}}$ in beige superimposed on the predicted structure of $\mathrm{Tfib}_{\text {Shamrock }}$ in blue. Red arrow shows where fusion occurs (residues 703, 704) between $\mathrm{Tfib}_{\mathrm{P} 1 \mathrm{~S}}$ and $\mathrm{Tfib}_{\mathrm{sfMu}}$ that was used to form fusion protein $\mathrm{Tfib}_{\text {shamrock. }} \mathrm{A}$ high amount of similarity can be seen with high variability in the variable loops. Structure prediction for other tail fiber fusions were attempted, but all had poor resolution and are not shown. 
Table S1 Plasmids used in this study

\begin{tabular}{|c|c|c|c|}
\hline Name & Source & Description & Sequence \\
\hline pRE107 & ATCC $^{\circledast}$ & ATCC $^{\circledR} 87671^{\text {TM }}$ Allelic exchange vector & ATCC $^{\circledR} 87691^{\text {TM }}$ \\
\hline p5014 & This study & Plasmid for deletion of P1 pacAB & GenBank MW263064 \\
\hline pKM208 & Murphy et al. & Lambda RED Recombineering plasmid & $\mathrm{N} / \mathrm{A}$ \\
\hline pUC19 & NEB & E. coli origin source & GenBank M77789.2 \\
\hline pUCP20 & Shanks et al. & pRO1600 origin source & GenBank U07165.1 \\
\hline pSD02 & Roche & reporter & GenBank MW263053 \\
\hline p2118 & Roche & pReporter & GenBank MW263054 \\
\hline p5010 & Roche & pReporter & GenBank MW263055 \\
\hline p5067 & This study & Tfib $_{\mathrm{p} 15 \mathrm{bSvo}}-$ Tfa $_{\mathrm{p} 15 \mathrm{~B}}$ TFDV "Tangerine" & GenBank MW263056 \\
\hline p5061 & This study & $\begin{array}{c}\text { Tfib }_{\text {P15703sfMus' }} \text { (hyb)-Tfa } \\
\text { "Shamu TFDV } \\
\text { "Shack" }\end{array}$ & GenBank MW263057 \\
\hline p5445 & This study & $\begin{array}{l}\text { Tfib }_{\text {P1S453-AJs10005 }}(\text { hyb) -Tfa } \\
\text { "Jazs10004 TFDV } \\
\text { "Jazzerry" }\end{array}$ & GenBank MW263058 \\
\hline p5446 & This study & $\begin{array}{l}\text { Tfib }_{\text {P1S502-WP_001000784 }}(\text { hyb) -Tfa } \\
\text { TFDV "Orchid" }\end{array}$ & GenBank MW263059 \\
\hline p5199 & This study & $\begin{array}{l}\text { Tfib }_{\text {P15214-Ec16437 }}\left(\text { hyb) }- \text { Tfa }_{\text {Ec16437 }} \text { TFDV }\right. \\
\text { "Banana" }\end{array}$ & GenBank MW263060 \\
\hline p5372 & This study & $\begin{array}{l}\text { Tfib }_{\text {P1S214-Sms0004 }}\left(\text { hyb) }- \text { Tfa }_{\text {Sms0004 }} \text { TFDV }\right. \\
\text { "Gold" }\end{array}$ & GenBank MW263061 \\
\hline p5379 & This study & $\begin{array}{c}\text { Tfib }_{\text {P15214-Pms15785 }}(\mathrm{hyb})-\mathrm{Tfa}_{\mathrm{Pms} 15785} \text { TFDV } \\
\text { "Rio" }\end{array}$ & GenBank MW263062 \\
\hline P5045 & This study & Tfib $_{P 1 S}-T_{P a} a_{P 1 S}$ TFDV "P1 S" & GenBank MW263063 \\
\hline p5027 & This study & pReporter v1.1 & GenBank MW263049 \\
\hline p5028 & This study & pReporter v1.2 & GenBank MW263050 \\
\hline p5222 & This study & pReporter v1.3 & GenBank MW263052 \\
\hline p5231 & This study & pReporter v2.2 & GenBank MW263051 \\
\hline
\end{tabular}

Legend - New England Biolabs (NEB) 
Table S2 Smarticles production strains and cloning strains used in this study

Name Source Description

\begin{tabular}{|c|c|c|}
\hline NEB5 $\alpha$ & $\begin{array}{l}\text { New England } \\
\text { Biolabs }\end{array}$ & $\begin{array}{l}\text { E. coli cloning strain for plasmid } \\
\text { propagation (NEB, C2987I) }\end{array}$ \\
\hline $\begin{array}{l}\text { TransforMax } \\
\text { EC100D }^{\mathrm{TM}} \text { pir-116 }\end{array}$ & Lucigen & $\begin{array}{c}\text { E. coli cloning strain with pir gene for } \\
\text { stable replication of R6Kpori containing } \\
\text { plasmid }\end{array}$ \\
\hline BAA-1001 & ATCC & $\begin{array}{l}\text { Phage P1 mod749::IS5 cl-100, a lysogentic } \\
\text { phage present in E. coli (ATCC BAA-1001) }\end{array}$ \\
\hline SEG_170 & In-house & $\begin{array}{c}\text { Strain for expressing TFDVs. P1 } \\
\text { mod749::IS5 cl-100 pacAB::HygR and s- } \\
\text { u::aadA }\end{array}$ \\
\hline SEG_281 & In-house & $\begin{array}{c}\text { Strain genomic expression } \\
\text { Recombineering. P1 mod749::IS5 cl-100 } \\
\triangle \text { pacAB and s-u::aadA + pKM208 }\end{array}$ \\
\hline SEG_172 & In-house & $\begin{array}{c}\text { "Complemented Chassis" SEG_170 + p5045 } \\
\text { Tfib }_{\text {P1s-Tfa }}-\text { Tf }_{\text {p }} \text { TFDV }\end{array}$ \\
\hline SEG_595 & In-house & $\begin{array}{c}\text { “Unmodified Chassis" Phage P1 } \\
\text { mod749::IS5 cl-100,a lysogentic phage } \\
\text { present E. coli (ATCC BAA-1001) } \\
\text { packaging line with } \triangle \text { pacAB + p5010 } \\
\text { pReporter }\end{array}$ \\
\hline SEG_719 & In-house & "Bald Chassis" SEG_170 + p5028 pReporter \\
\hline SEG_240 & In-house & SEG_170 + p5067 Tangerine TFDV \\
\hline SEG_242 & In-house & SEG_170 + p5061 Shamrock TFDV \\
\hline SEG_317 & In-house & SEG_170 + p5445 Jazzberry TFDV \\
\hline SEG_476 & In-house & SEG_170 + p5446 Orchid TFDV \\
\hline SEG_535 & In-house & SEG_170 + p5199 Banana TFDV \\
\hline SEG_737 & In-house & SEG_170 + p5372 Gold TFDV \\
\hline SEG_769 & In-house & SEG_170 + p5379 Rio TFDV \\
\hline SEG_307 & In-house & Genomic Shamrock + p5027 pReporter \\
\hline SEG_374 & In-house & Genomic Jazzberry + p5027 pReporter \\
\hline SEG_632 & In-house & Genomic Banana + p5222 pReporter \\
\hline SEG_633 & In-house & Genomic Tangerine + p5231 pReporter \\
\hline SEG_830 & In-house & Genomic Rio + p5222 pReporter \\
\hline SEG_835 & In-house & Genomic Gold + p5222 pReporter \\
\hline SEG_631 & In-house & Genomic ShamJazz + p5222 pReporter \\
\hline
\end{tabular}


E. cloacae strain for Tfib and Tfa Banana source

Sms0004

Roche

S. marcescens strain for Tfib and Tfa Gold source 
Table S3 Strains used in Inclusivity and Exclusivity Evaluation

\begin{tabular}{|c|c|c|c|c|c|c|}
\hline $\begin{array}{l}\text { Strain } \\
\text { Number }\end{array}$ & Panel & Genus & Species & RMSCC & Source & Source ID \\
\hline 1 & E. coli & Escherichia & coli & 03075 & ATCC & 25922 \\
\hline 2 & E. coli & Escherichia & coli & 15488 & IHMA & 1275635 \\
\hline 3 & E. coli & Escherichia & coli & 16213 & CDC & AR-0069 \\
\hline 4 & E. coli & Escherichia & coli & 16214 & CDC & AR-0061 \\
\hline 5 & E. coli & Escherichia & coli & 16215 & CDC & AR-0055 \\
\hline 6 & E. coli & Escherichia & coli & 16216 & $\mathrm{CDC}$ & AR-0048 \\
\hline 7 & E. coli & Escherichia & coli & 16217 & CDC & AR-0006 \\
\hline 8 & E. coli & Escherichia & coli & 16219 & $\mathrm{CDC}$ & AR-0114 \\
\hline 9 & E. coli & Escherichia & coli & 16220 & CDC & AR-0162 \\
\hline 10 & E. coli & Escherichia & coli & 16221 & $\mathrm{CDC}$ & AR-0150 \\
\hline 11 & E. coli & Escherichia & coli & 16223 & CDC & AR-0151 \\
\hline 12 & E. coli & Escherichia & coli & 16224 & CDC & AR-0118 \\
\hline 13 & E. coli & Escherichia & coli & 16226 & CDC & AR-0119 \\
\hline 14 & E. coli & Escherichia & coli & 16227 & $\mathrm{CDC}$ & AR-0149 \\
\hline 15 & E. coli & Escherichia & coli & 16228 & $\mathrm{CDC}$ & AR-0128 \\
\hline 16 & E. coli & Escherichia & coli & 16444 & IHMA & 449256 \\
\hline 17 & E. coli & Escherichia & coli & 16445 & IHMA & 449272 \\
\hline 18 & E. coli & Escherichia & coli & 16446 & IHMA & 449334 \\
\hline 19 & E. coli & Escherichia & coli & 16449 & IHMA & 581348 \\
\hline 20 & E. coli & Escherichia & coli & 16450 & IHMA & 581480 \\
\hline 21 & E. coli & Escherichia & coli & 16452 & IHMA & 581817 \\
\hline 22 & E. coli & Escherichia & coli & 16454 & IHMA & 586030 \\
\hline 23 & E. coli & Escherichia & coli & 16456 & IHMA & 605879 \\
\hline
\end{tabular}




\begin{tabular}{|c|c|c|c|c|c|c|}
\hline 24 & E. coli & Escherichia & coli & 16457 & IHMA & 664507 \\
\hline 25 & E. coli & Escherichia & coli & 16458 & IHMA & 664520 \\
\hline 26 & E. coli & Escherichia & coli & 16459 & IHMA & 664523 \\
\hline 27 & E. coli & Escherichia & coli & 16460 & IHMA & 664528 \\
\hline 28 & E. coli & Escherichia & coli & 16461 & IHMA & 684850 \\
\hline 29 & E. coli & Escherichia & coli & 16463 & IHMA & 734399 \\
\hline 30 & E. coli & Escherichia & coli & 16464 & IHMA & 768053 \\
\hline 31 & E. coli & Escherichia & coli & 16466 & IHMA & 786898 \\
\hline 32 & E. coli & Escherichia & coli & 16467 & IHMA & 787033 \\
\hline 33 & E. coli & Escherichia & coli & 16468 & IHMA & 787112 \\
\hline 34 & E. coli & Escherichia & coli & 16469 & IHMA & 787437 \\
\hline 35 & E. coli & Escherichia & coli & 16470 & ATCC & BAA-2452 \\
\hline 36 & E. coli & Escherichia & coli & 16472 & IHMA & 868726 \\
\hline 37 & E. coli & Escherichia & coli & 16473 & IHMA & 805459 \\
\hline 38 & E. coli & Escherichia & coli & 16474 & IHMA & 853090 \\
\hline 39 & E. coli & Escherichia & coli & 16475 & IHMA & 926425 \\
\hline 40 & E. coli & Escherichia & coli & 16476 & IHMA & 951011 \\
\hline 41 & E. coli & Escherichia & coli & 16477 & IHMA & 1093964 \\
\hline 42 & E. coli & Escherichia & coli & 16478 & IHMA & 1172732 \\
\hline 43 & E. coli & Escherichia & coli & 16479 & IHMA & 778861 \\
\hline 44 & E. coli & Escherichia & coli & 16480 & IHMA & 1104276 \\
\hline 45 & E. coli & Escherichia & coli & 16481 & IHMA & 820015 \\
\hline 46 & E. coli & Escherichia & coli & 16482 & IHMA & 787042 \\
\hline 47 & E. coli & Escherichia & coli & 16483 & IHMA & 1154134 \\
\hline 48 & E. coli & Escherichia & coli & 16484 & IHMA & 664526 \\
\hline 49 & E. coli & Escherichia & coli & 16485 & IHMA & 1156982 \\
\hline 50 & E. coli & Escherichia & coli & 16486 & IHMA & 1207319 \\
\hline 51 & E. coli & Escherichia & coli & 16495 & IHMA & 1371539 \\
\hline 52 & E. coli & Escherichia & coli & 16499 & IHMA & 1427416 \\
\hline 53 & E. coli & Escherichia & coli & 16501 & IHMA & 1435372 \\
\hline 54 & E. coli & Escherichia & coli & 16517 & IHMA & 600277 \\
\hline
\end{tabular}




\begin{tabular}{|c|c|c|c|c|c|c|}
\hline 55 & E. coli & Escherichia & coli & 16518 & IHMA & 850557 \\
\hline 56 & E. coli & Escherichia & coli & 16519 & IHMA & 855914 \\
\hline 57 & E. coli & Escherichia & coli & 16520 & IHMA & 856619 \\
\hline 58 & E. coli & Escherichia & coli & 16521 & IHMA & 867247 \\
\hline 59 & E. coli & Escherichia & coli & 16522 & IHMA & 868481 \\
\hline 60 & E. coli & Escherichia & coli & 16523 & IHMA & 883213 \\
\hline 61 & E. coli & Escherichia & coli & 16524 & IHMA & 884259 \\
\hline 62 & E. coli & Escherichia & coli & 16525 & IHMA & 929343 \\
\hline 63 & E. coli & Escherichia & coli & 16527 & IHMA & 963230 \\
\hline 64 & E. coli & Escherichia & coli & 16528 & IHMA & 968892 \\
\hline 65 & E. coli & Escherichia & coli & 16529 & IHMA & 1007517 \\
\hline 66 & E. coli & Escherichia & coli & 16530 & ATCC & 35218 \\
\hline 67 & E. coli & Escherichia & coli & 16533 & ATCC & 700926 \\
\hline 68 & E. coli & Escherichia & coli & 16538 & IHMA & 855483 \\
\hline 69 & E. coli & Escherichia & coli & 16539 & IHMA & 866992 \\
\hline 70 & E. coli & Escherichia & coli & 16540 & IHMA & 872188 \\
\hline 71 & E. coli & Escherichia & coli & 16541 & IHMA & 883184 \\
\hline 72 & E. coli & Escherichia & coli & 16542 & IHMA & 901128 \\
\hline 73 & E. coli & Escherichia & coli & 16543 & IHMA & 924332 \\
\hline 74 & E. coli & Escherichia & coli & 16544 & IHMA & 925051 \\
\hline 75 & E. coli & Escherichia & coli & 16545 & IHMA & 949372 \\
\hline 76 & E. coli & Escherichia & coli & 16546 & IHMA & 981491 \\
\hline 77 & E. coli & Escherichia & coli & 16547 & IHMA & 984046 \\
\hline 78 & E. coli & Escherichia & coli & 16548 & IHMA & 991849 \\
\hline 79 & E. coli & Escherichia & coli & 16549 & ATCC & 25404 \\
\hline 80 & E. coli & Escherichia & coli & 16550 & ATCC & BAA-2523 \\
\hline 81 & E. coli & Escherichia & coli & 16551 & IHMA & 845014 \\
\hline 82 & E. coli & Escherichia & coli & 16552 & IHMA & 885463 \\
\hline 83 & E. coli & Escherichia & coli & 16553 & IHMA & 957452 \\
\hline 84 & E. coli & Escherichia & coli & 16554 & $\mathrm{CDC}$ & AR-0089 \\
\hline 85 & E. coli & Escherichia & coli & 16555 & $\mathrm{CDC}$ & AR-0081 \\
\hline
\end{tabular}




\begin{tabular}{|c|c|c|c|c|c|c|}
\hline 86 & E. coli & Escherichia & coli & 16556 & $\mathrm{CDC}$ & AR-0086 \\
\hline 87 & E. coli & Escherichia & coli & 16557 & $\mathrm{CDC}$ & AR-0058 \\
\hline 88 & E. coli & Escherichia & coli & 16558 & $\mathrm{CDC}$ & AR-0077 \\
\hline 89 & E. coli & Escherichia & coli & 16559 & $\mathrm{CDC}$ & AR-0084 \\
\hline 1 & K. pneumoniae & Klebsiella & pneumoniae & Kpn0167 & ATCC & BAA-1898 \\
\hline 2 & K. pneumoniae & Klebsiella & pneumoniae & Kpn0233 & IHMA & 827112 \\
\hline 3 & K. pneumoniae & Klebsiella & pneumoniae & Kpn0234 & IHMA & 827114 \\
\hline 4 & K. pneumoniae & Klebsiella & pneumoniae & Kpn0420 & IHMA & 1557658 \\
\hline 5 & K. pneumoniae & Klebsiella & pneumoniae & Kpn0421 & IHMA & 1557642 \\
\hline 6 & K. pneumoniae & Klebsiella & pneumoniae & Kpn0466 & IHMA & 1700845 \\
\hline 7 & K. pneumoniae & Klebsiella & pneumoniae & Kpn0467 & IHMA & 1676587 \\
\hline 8 & K. pneumoniae & Klebsiella & pneumoniae & Kpn0468 & IHMA & 1580208 \\
\hline 9 & K. pneumoniae & Klebsiella & pneumoniae & Kpn0469 & IHMA & 1660461 \\
\hline 10 & K. pneumoniae & Klebsiella & pneumoniae & Kpn0470 & IHMA & 1677437 \\
\hline 11 & K. pneumoniae & Klebsiella & pneumoniae & Kpn0471 & IHMA & 1658630 \\
\hline 12 & K. pneumoniae & Klebsiella & pneumoniae & Kpn0472 & IHMA & 1610259 \\
\hline 13 & K. pneumoniae & Klebsiella & pneumoniae & Kpn0473 & IHMA & 1598062 \\
\hline 14 & K. pneumoniae & Klebsiella & pneumoniae & Kpn0474 & IHMA & 1621878 \\
\hline 15 & K. pneumoniae & Klebsiella & pneumoniae & Kpn0475 & IHMA & 1621883 \\
\hline 16 & K. pneumoniae & Klebsiella & pneumoniae & Kpn0476 & IHMA & 1648094 \\
\hline 17 & K. pneumoniae & Klebsiella & pneumoniae & Kpn0477 & IHMA & 1691410 \\
\hline 18 & K. pneumoniae & Klebsiella & pneumoniae & 16229 & $\mathrm{CDC}$ & AR-0109 \\
\hline 19 & K. pneumoniae & Klebsiella & pneumoniae & 16232 & $\mathrm{CDC}$ & AR-0106 \\
\hline 20 & K. pneumoniae & Klebsiella & pneumoniae & 16234 & $\mathrm{CDC}$ & AR-0066 \\
\hline 21 & K. pneumoniae & Klebsiella & pneumoniae & 16235 & $\mathrm{CDC}$ & AR-0040 \\
\hline 22 & K. pneumoniae & Klebsiella & pneumoniae & 16236 & $\mathrm{CDC}$ & AR-0068 \\
\hline 23 & K. pneumoniae & Klebsiella & pneumoniae & 16238 & $\mathrm{CDC}$ & AR-0075 \\
\hline 24 & K. pneumoniae & Klebsiella & pneumoniae & 16239 & $\mathrm{CDC}$ & AR-0047 \\
\hline 25 & K. pneumoniae & Klebsiella & pneumoniae & 16241 & $\mathrm{CDC}$ & AR-0046 \\
\hline 26 & K. pneumoniae & Klebsiella & pneumoniae & 16243 & $\mathrm{CDC}$ & AR-0043 \\
\hline 27 & K. pneumoniae & Klebsiella & pneumoniae & 16245 & $\mathrm{CDC}$ & AR-0041 \\
\hline
\end{tabular}




\begin{tabular}{|c|c|c|c|c|c|c|}
\hline 28 & K. pneumoniae & Klebsiella & pneumoniae & 16248 & $\mathrm{CDC}$ & AR-0005 \\
\hline 29 & K. pneumoniae & Klebsiella & pneumoniae & 16250 & $\mathrm{CDC}$ & AR-0010 \\
\hline 30 & K. pneumoniae & Klebsiella & pneumoniae & 16253 & $\mathrm{CDC}$ & AR-0139 \\
\hline 31 & K. pneumoniae & Klebsiella & pneumoniae & 16256 & $\mathrm{CDC}$ & AR-0113 \\
\hline 32 & K. pneumoniae & Klebsiella & pneumoniae & 16261 & $\mathrm{CDC}$ & AR-0148 \\
\hline 33 & K. pneumoniae & Klebsiella & pneumoniae & 16262 & $\mathrm{CDC}$ & AR-0112 \\
\hline 34 & K. pneumoniae & Klebsiella & pneumoniae & 16264 & $\mathrm{CDC}$ & AR-0140 \\
\hline 35 & K. pneumoniae & Klebsiella & pneumoniae & 16265 & $\mathrm{CDC}$ & AR-0138 \\
\hline 36 & K. pneumoniae & Klebsiella & pneumoniae & 16266 & $\mathrm{CDC}$ & AR-0160 \\
\hline 37 & K. pneumoniae & Klebsiella & pneumoniae & 16268 & $\mathrm{CDC}$ & AR-0120 \\
\hline 38 & K. pneumoniae & Klebsiella & pneumoniae & 16272 & $\mathrm{CDC}$ & AR-0129 \\
\hline 39 & K. pneumoniae & Klebsiella & pneumoniae & 16560 & IHMA & 468861 \\
\hline 40 & K. pneumoniae & Klebsiella & pneumoniae & 16561 & IHMA & 469384 \\
\hline 41 & K. pneumoniae & Klebsiella & pneumoniae & 16563 & IHMA & 683051 \\
\hline 42 & K. pneumoniae & Klebsiella & pneumoniae & 16564 & IHMA & 718993 \\
\hline 43 & K. pneumoniae & Klebsiella & pneumoniae & 16565 & IHMA & 720976 \\
\hline 44 & K. pneumoniae & Klebsiella & pneumoniae & 16566 & IHMA & 722861 \\
\hline 45 & K. pneumoniae & Klebsiella & pneumoniae & 16567 & IHMA & 749890 \\
\hline 46 & K. pneumoniae & Klebsiella & pneumoniae & 16568 & IHMA & 752288 \\
\hline 47 & K. pneumoniae & Klebsiella & pneumoniae & 16576 & IHMA & 450259 \\
\hline 48 & K. pneumoniae & Klebsiella & pneumoniae & 16577 & IHMA & 451135 \\
\hline 49 & K. pneumoniae & Klebsiella & pneumoniae & 16578 & IHMA & 451166 \\
\hline 50 & K. pneumoniae & Klebsiella & pneumoniae & 16580 & IHMA & 516426 \\
\hline 51 & K. pneumoniae & Klebsiella & pneumoniae & 16583 & IHMA & 644641 \\
\hline 52 & K. pneumoniae & Klebsiella & pneumoniae & 16584 & IHMA & 648540 \\
\hline 53 & K. pneumoniae & Klebsiella & pneumoniae & 16585 & IHMA & 684840 \\
\hline 54 & K. pneumoniae & Klebsiella & pneumoniae & 16586 & IHMA & 685215 \\
\hline 55 & K. pneumoniae & Klebsiella & pneumoniae & 16587 & IHMA & 732789 \\
\hline 56 & K. pneumoniae & Klebsiella & pneumoniae & 16589 & IHMA & 735975 \\
\hline 57 & K. pneumoniae & Klebsiella & pneumoniae & 16590 & IHMA & 768140 \\
\hline 58 & K. pneumoniae & Klebsiella & pneumoniae & 16591 & IHMA & 780502 \\
\hline
\end{tabular}




\begin{tabular}{|c|c|c|c|c|c|c|}
\hline 59 & K. pneumoniae & Klebsiella & pneumoniae & 16592 & IHMA & 787114 \\
\hline 60 & K. pneumoniae & Klebsiella & pneumoniae & 16594 & IHMA & 449170 \\
\hline 61 & K. pneumoniae & Klebsiella & pneumoniae & 16595 & $\mathrm{CDC}$ & AR-0107 \\
\hline 62 & K. pneumoniae & Klebsiella & pneumoniae & 16629 & IHMA & 856271 \\
\hline 63 & K. pneumoniae & Klebsiella & pneumoniae & 16631 & IHMA & 866200 \\
\hline 64 & K. pneumoniae & Klebsiella & pneumoniae & 16632 & IHMA & 868500 \\
\hline 65 & K. pneumoniae & Klebsiella & pneumoniae & 16633 & IHMA & 871490 \\
\hline 66 & K. pneumoniae & Klebsiella & pneumoniae & 16634 & IHMA & 890148 \\
\hline 67 & K. pneumoniae & Klebsiella & pneumoniae & 16635 & IHMA & 921251 \\
\hline 68 & K. pneumoniae & Klebsiella & pneumoniae & 16636 & IHMA & 921267 \\
\hline 69 & K. pneumoniae & Klebsiella & pneumoniae & 16638 & IHMA & 949823 \\
\hline 70 & K. pneumoniae & Klebsiella & pneumoniae & 16639 & IHMA & 951034 \\
\hline 71 & K. pneumoniae & Klebsiella & pneumoniae & 16640 & IHMA & 961841 \\
\hline 72 & K. pneumoniae & Klebsiella & pneumoniae & 16641 & IHMA & 972048 \\
\hline 73 & K. pneumoniae & Klebsiella & pneumoniae & 16642 & IHMA & 1036631 \\
\hline 74 & K. pneumoniae & Klebsiella & pneumoniae & 16643 & IHMA & 1043255 \\
\hline 75 & K. pneumoniae & Klebsiella & pneumoniae & 16644 & $\mathrm{CDC}$ & AR-0079 \\
\hline 76 & K. pneumoniae & Klebsiella & pneumoniae & 16645 & $\mathrm{CDC}$ & AR-0016 \\
\hline 77 & K. pneumoniae & Klebsiella & pneumoniae & 16646 & $\mathrm{CDC}$ & AR-0012 \\
\hline 78 & K. pneumoniae & Klebsiella & pneumoniae & 16647 & ATCC & 23356 \\
\hline 79 & K. pneumoniae & Klebsiella & pneumoniae & 16648 & $\mathrm{CDC}$ & AR-0087 \\
\hline 80 & K. pneumoniae & Klebsiella & pneumoniae & 16649 & IHMA & 586246 \\
\hline 81 & K. pneumoniae & Klebsiella & pneumoniae & 16650 & IHMA & 598865 \\
\hline 82 & K. pneumoniae & Klebsiella & pneumoniae & 16651 & IHMA & 847363 \\
\hline 83 & K. pneumoniae & Klebsiella & pneumoniae & 16652 & IHMA & 883234 \\
\hline 84 & K. pneumoniae & Klebsiella & pneumoniae & 16653 & IHMA & 918237 \\
\hline 85 & K. pneumoniae & Klebsiella & pneumoniae & 16978 & ATCC & 700603 \\
\hline 86 & K. pneumoniae & Klebsiella & pneumoniae & 17202 & IHMA & 850711 \\
\hline 87 & K. pneumoniae & Klebsiella & pneumoniae & 17203 & IHMA & 994043 \\
\hline 1 & E. cloacae & Enterobacter & cloacae & Ecl0037 & IHMA & 1556620 \\
\hline 2 & E. cloacae & Enterobacter & cloacae & Ecl0038 & IHMA & 1556617 \\
\hline
\end{tabular}




\begin{tabular}{|c|c|c|c|c|c|}
\hline E. cloacae & Enterobacter & cloacae & Ecl0039 & IHMA & 1556618 \\
\hline E. cloacae & Enterobacter & cloacae & Ecl0040 & IHMA & 1583088 \\
\hline E. cloacae & Enterobacter & cloacae & Ecl0041 & IHMA & 1555907 \\
\hline E. cloacae & Enterobacter & cloacae & Ecl0042 & IHMA & 1559438 \\
\hline E. cloacae & Enterobacter & cloacae & Ecl0043 & IHMA & 1590179 \\
\hline E. cloacae & Enterobacter & cloacae & Ecl0044 & IHMA & 1572134 \\
\hline E. cloacae & Enterobacter & cloacae & Ecl0045 & IHMA & 1639322 \\
\hline E. cloacae & Enterobacter & cloacae & Ecl0046 & IHMA & 1612749 \\
\hline E. cloacae & Enterobacter & cloacae & Ecl0047 & IHMA & 1608083 \\
\hline E. cloacae & Enterobacter & cloacae & Ecl0048 & IHMA & 1608094 \\
\hline E. cloacae & Enterobacter & cloacae & Ecl0049 & IHMA & 1608097 \\
\hline E. cloacae & Enterobacter & cloacae & Ecl0050 & IHMA & 1680471 \\
\hline E. cloacae & Enterobacter & cloacae & Ecl0051 & IHMA & 1683088 \\
\hline E. cloacae & Enterobacter & cloacae & Ecl0052 & IHMA & 1656078 \\
\hline E. cloacae & Enterobacter & cloacae & Ecl0053 & IHMA & 1650083 \\
\hline E. cloacae & Enterobacter & cloacae & Ecl0054 & IHMA & 1650090 \\
\hline E. cloacae & Enterobacter & cloacae & 15430 & IHMA & 1062735 \\
\hline E. cloacae & Enterobacter & cloacae & 15431 & IHMA & 1062774 \\
\hline E. cloacae & Enterobacter & cloacae & 15440 & IHMA & 1082097 \\
\hline E. cloacae & Enterobacter & cloacae & 15441 & IHMA & 1083806 \\
\hline E. cloacae & Enterobacter & cloacae & 15442 & IHMA & 1089036 \\
\hline E. cloacae & Enterobacter & cloacae & 15444 & IHMA & 1114095 \\
\hline E. cloacae & Enterobacter & cloacae & 15448 & IHMA & 1184909 \\
\hline E. cloacae & Enterobacter & cloacae & 15450 & IHMA & 1207294 \\
\hline E. cloacae & Enterobacter & cloacae & 15451 & IHMA & 1207298 \\
\hline E. cloacae & Enterobacter & cloacae & 15452 & IHMA & 1207303 \\
\hline E. cloacae & Enterobacter & cloacae & 15453 & IHMA & 1207309 \\
\hline E. cloacae & Enterobacter & cloacae & 15454 & IHMA & 1207332 \\
\hline E. cloacae & Enterobacter & cloacae & 15455 & IHMA & 1207444 \\
\hline E. cloacae & Enterobacter & cloacae & 15456 & IHMA & 1207515 \\
\hline E. cloacae & Enterobacter & cloacae & 15457 & IHMA & 1207979 \\
\hline
\end{tabular}




\begin{tabular}{|c|c|c|c|c|c|c|}
\hline 34 & E. cloacae & Enterobacter & cloacae & 15462 & IHMA & 1212332 \\
\hline 35 & E. cloacae & Enterobacter & cloacae & 15464 & IHMA & 1214824 \\
\hline 36 & E. cloacae & Enterobacter & cloacae & 15467 & IHMA & 1219025 \\
\hline 37 & E. cloacae & Enterobacter & cloacae & 15473 & IHMA & 1223644 \\
\hline 38 & E. cloacae & Enterobacter & cloacae & 15474 & IHMA & 1223660 \\
\hline 39 & E. cloacae & Enterobacter & cloacae & 15475 & IHMA & 1224128 \\
\hline 40 & E. cloacae & Enterobacter & cloacae & 15476 & IHMA & 1229259 \\
\hline 41 & E. cloacae & Enterobacter & cloacae & 15477 & IHMA & 1229294 \\
\hline 42 & E. cloacae & Enterobacter & cloacae & 15478 & IHMA & 1229468 \\
\hline 43 & E. cloacae & Enterobacter & cloacae & 15479 & IHMA & 1229469 \\
\hline 44 & E. cloacae & Enterobacter & cloacae & 15480 & IHMA & 1232477 \\
\hline 45 & E. cloacae & Enterobacter & cloacae & 15481 & IHMA & 1240717 \\
\hline 46 & E. cloacae & Enterobacter & cloacae & 15482 & IHMA & 1245555 \\
\hline 47 & E. cloacae & Enterobacter & cloacae & 15483 & IHMA & 1247103 \\
\hline 48 & E. cloacae & Enterobacter & cloacae & 15485 & IHMA & 1248855 \\
\hline 49 & E. cloacae & Enterobacter & cloacae & 15486 & IHMA & 1255416 \\
\hline 50 & E. cloacae & Enterobacter & cloacae & 15492 & IHMA & 1294802 \\
\hline 51 & E. cloacae & Enterobacter & cloacae & 15494 & IHMA & 1308607 \\
\hline 52 & E. cloacae & Enterobacter & cloacae & 15495 & IHMA & 1316136 \\
\hline 53 & E. cloacae & Enterobacter & cloacae & 15496 & IHMA & 1317130 \\
\hline 54 & E. cloacae & Enterobacter & cloacae & 15497 & IHMA & 1318534 \\
\hline 55 & E. cloacae & Enterobacter & cloacae & 15498 & IHMA & 1324577 \\
\hline 56 & E. cloacae & Enterobacter & cloacae & 15499 & IHMA & 1324619 \\
\hline 57 & E. cloacae & Enterobacter & cloacae & 15654 & ATCC & BAA-234 \\
\hline 58 & E. cloacae & Enterobacter & cloacae & 16274 & $\mathrm{CDC}$ & AR-0002 \\
\hline 59 & E. cloacae & Enterobacter & cloacae & 16275 & $\mathrm{CDC}$ & AR-0008 \\
\hline 60 & E. cloacae & Enterobacter & cloacae & 16276 & $\mathrm{CDC}$ & AR-0032 \\
\hline 61 & E. cloacae & Enterobacter & cloacae & 16277 & $\mathrm{CDC}$ & AR-0038 \\
\hline 62 & E. cloacae & Enterobacter & cloacae & 16311 & $\mathrm{CDC}$ & AR-0053 \\
\hline 63 & E. cloacae & Enterobacter & cloacae & 16312 & $\mathrm{CDC}$ & AR-0072 \\
\hline 64 & E. cloacae & Enterobacter & cloacae & 16313 & $\mathrm{CDC}$ & AR-0093 \\
\hline
\end{tabular}




\begin{tabular}{|c|c|c|c|c|c|c|}
\hline 65 & E. cloacae & Enterobacter & cloacae & 16314 & $\mathrm{CDC}$ & AR-0154 \\
\hline 66 & E. cloacae & Enterobacter & cloacae & 16315 & $\mathrm{CDC}$ & AR-0163 \\
\hline 67 & E. cloacae & Enterobacter & cloacae & 16316 & $\mathrm{CDC}$ & AR-0164 \\
\hline 68 & E. cloacae & Enterobacter & cloacae & 16339 & IHMA & 1525454 \\
\hline 69 & E. cloacae & Enterobacter & cloacae & 16424 & IHMA & 775952 \\
\hline 70 & E. cloacae & Enterobacter & cloacae & 16425 & IHMA & 855980 \\
\hline 71 & E. cloacae & Enterobacter & cloacae & 16426 & IHMA & 893469 \\
\hline 72 & E. cloacae & Enterobacter & cloacae & 16427 & IHMA & 936177 \\
\hline 73 & E. cloacae & Enterobacter & cloacae & 16428 & IHMA & 969526 \\
\hline 74 & E. cloacae & Enterobacter & cloacae & 16429 & IHMA & 951081 \\
\hline 75 & E. cloacae & Enterobacter & cloacae & 16430 & IHMA & 906903 \\
\hline 76 & E. cloacae & Enterobacter & cloacae & 16432 & IHMA & 779131 \\
\hline 77 & E. cloacae & Enterobacter & cloacae & 16433 & IHMA & 822494 \\
\hline 78 & E. cloacae & Enterobacter & cloacae & 16434 & IHMA & 910623 \\
\hline 79 & E. cloacae & Enterobacter & cloacae & 16435 & IHMA & 946148 \\
\hline 80 & E. cloacae & Enterobacter & cloacae & 16438 & IHMA & 945751 \\
\hline 81 & E. cloacae & Enterobacter & cloacae & 16439 & IHMA & 963056 \\
\hline 82 & E. cloacae & Enterobacter & cloacae & 16440 & IHMA & 1001462 \\
\hline 83 & E. cloacae & Enterobacter & cloacae & 16441 & IHMA & 942523 \\
\hline 84 & E. cloacae & Enterobacter & cloacae & 16442 & $\mathrm{CDC}$ & AR-0060 \\
\hline 85 & E. cloacae & Enterobacter & cloacae & 16885 & IHMA & 1466599 \\
\hline 86 & E. cloacae & Enterobacter & cloacae & 17412 & $\mathrm{CDC}$ & AR-0073 \\
\hline 87 & E. cloacae & Enterobacter & cloacae & 17435 & $\mathrm{CDC}$ & AR-0136 \\
\hline 1 & P. mirabilis & Proteus & mirabilis & Pms0002 & IHMA & 773649 \\
\hline 2 & P. mirabilis & Proteus & mirabilis & Pms0003 & IHMA & 751906 \\
\hline 3 & P. mirabilis & Proteus & mirabilis & Pms0004 & IHMA & 850377 \\
\hline 4 & P. mirabilis & Proteus & mirabilis & Pms0006 & IHMA & 855991 \\
\hline 5 & P. mirabilis & Proteus & mirabilis & Pms0007 & IHMA & 832136 \\
\hline 6 & P. mirabilis & Proteus & mirabilis & Pms0008 & IHMA & 871552 \\
\hline 7 & P. mirabilis & Proteus & mirabilis & Pms0010 & IHMA & 889600 \\
\hline 8 & P. mirabilis & Proteus & mirabilis & Pms0011 & IHMA & 862386 \\
\hline
\end{tabular}




\begin{tabular}{|c|c|c|c|c|c|c|}
\hline 9 & P. mirabilis & Proteus & mirabilis & Pms0012 & IHMA & 862388 \\
\hline 10 & P. mirabilis & Proteus & mirabilis & Pms0013 & IHMA & 844637 \\
\hline 11 & P. mirabilis & Proteus & mirabilis & Pms0014 & IHMA & 924278 \\
\hline 12 & P. mirabilis & Proteus & mirabilis & Pms0016 & IHMA & 971507 \\
\hline 13 & P. mirabilis & Proteus & mirabilis & Pms0017 & Dynacare & 1 \\
\hline 14 & P. mirabilis & Proteus & mirabilis & Pms0018 & Dynacare & 2 \\
\hline 15 & P. mirabilis & Proteus & mirabilis & Pms0019 & Dynacare & 3 \\
\hline 16 & P. mirabilis & Proteus & mirabilis & Pms0020 & Dynacare & 4 \\
\hline 17 & P. mirabilis & Proteus & mirabilis & Pms0022 & Dynacare & 6 \\
\hline 18 & P. mirabilis & Proteus & mirabilis & Pms0023 & Dynacare & 7 \\
\hline 19 & P. mirabilis & Proteus & mirabilis & Pms0024 & Dynacare & 8 \\
\hline 20 & P. mirabilis & Proteus & mirabilis & Pms0025 & Dynacare & 10 \\
\hline 21 & P. mirabilis & Proteus & mirabilis & Pms0026 & Dynacare & 11 \\
\hline 22 & P. mirabilis & Proteus & mirabilis & 15780 & Roche & 721355 \\
\hline 23 & P. mirabilis & Proteus & mirabilis & 15781 & Roche & 716932 \\
\hline 24 & P. mirabilis & Proteus & mirabilis & 15782 & Roche & 402944 \\
\hline 25 & P. mirabilis & Proteus & mirabilis & 15783 & Roche & 713582 \\
\hline 26 & P. mirabilis & Proteus & mirabilis & 15784 & Roche & 604768 \\
\hline 27 & P. mirabilis & Proteus & mirabilis & 15785 & Roche & $703614-2$ \\
\hline 28 & P. mirabilis & Proteus & mirabilis & 15786 & Roche & 802700 \\
\hline 29 & P. mirabilis & Proteus & mirabilis & 15788 & Roche & 700198 \\
\hline 30 & P. mirabilis & Proteus & mirabilis & 15838 & IHMA & 1413117 \\
\hline 31 & P. mirabilis & Proteus & mirabilis & 15839 & IHMA & 1413946 \\
\hline 32 & P. mirabilis & Proteus & mirabilis & 15840 & IHMA & 1413949 \\
\hline 33 & P. mirabilis & Proteus & mirabilis & 15844 & IHMA & 1414324 \\
\hline 34 & P. mirabilis & Proteus & mirabilis & 15849 & IHMA & 1413951 \\
\hline 35 & P. mirabilis & Proteus & mirabilis & 15850 & IHMA & 1414434 \\
\hline 36 & P. mirabilis & Proteus & mirabilis & 15873 & IHMA & 1416988 \\
\hline 37 & P. mirabilis & Proteus & mirabilis & 15875 & IHMA & 1417558 \\
\hline 38 & P. mirabilis & Proteus & mirabilis & 15881 & IHMA & 1424504 \\
\hline 39 & P. mirabilis & Proteus & mirabilis & 15882 & IHMA & 1424505 \\
\hline
\end{tabular}




\begin{tabular}{|c|c|c|c|c|c|c|}
\hline 40 & P. mirabilis & Proteus & mirabilis & 15883 & IHMA & 1424506 \\
\hline 41 & P. mirabilis & Proteus & mirabilis & 15885 & IHMA & 1425024 \\
\hline 42 & P. mirabilis & Proteus & mirabilis & 15888 & IHMA & 1428989 \\
\hline 43 & P. mirabilis & Proteus & mirabilis & 15890 & IHMA & 1429294 \\
\hline 44 & P. mirabilis & Proteus & mirabilis & 15892 & IHMA & 1430131 \\
\hline 45 & P. mirabilis & Proteus & mirabilis & 15896 & IHMA & 1430423 \\
\hline 46 & P. mirabilis & Proteus & mirabilis & 15899 & IHMA & 1431845 \\
\hline 47 & P. mirabilis & Proteus & mirabilis & 15900 & IHMA & 1431846 \\
\hline 48 & P. mirabilis & Proteus & mirabilis & 15914 & IHMA & 1434790 \\
\hline 49 & P. mirabilis & Proteus & mirabilis & 15916 & IHMA & 1436270 \\
\hline 50 & P. mirabilis & Proteus & mirabilis & 15917 & IHMA & 1438982 \\
\hline 51 & P. mirabilis & Proteus & mirabilis & 15918 & IHMA & 1439864 \\
\hline 52 & P. mirabilis & Proteus & mirabilis & 15919 & IHMA & 1439866 \\
\hline 53 & P. mirabilis & Proteus & mirabilis & 15931 & IHMA & 1445808 \\
\hline 54 & P. mirabilis & Proteus & mirabilis & 15934 & IHMA & 1446346 \\
\hline 55 & P. mirabilis & Proteus & mirabilis & 15935 & IHMA & 1446347 \\
\hline 56 & P. mirabilis & Proteus & mirabilis & 15938 & IHMA & 1447990 \\
\hline 57 & P. mirabilis & Proteus & mirabilis & 15940 & IHMA & 1458278 \\
\hline 58 & P. mirabilis & Proteus & mirabilis & 15941 & IHMA & 1458651 \\
\hline 59 & P. mirabilis & Proteus & mirabilis & 15943 & IHMA & 1459067 \\
\hline 60 & P. mirabilis & Proteus & mirabilis & 15946 & IHMA & 1465491 \\
\hline 61 & P. mirabilis & Proteus & mirabilis & 15947 & IHMA & 1465492 \\
\hline 62 & P. mirabilis & Proteus & mirabilis & 15948 & IHMA & 1466714 \\
\hline 63 & P. mirabilis & Proteus & mirabilis & 15949 & IHMA & 1466794 \\
\hline 64 & P. mirabilis & Proteus & mirabilis & 15952 & IHMA & 1468357 \\
\hline 65 & P. mirabilis & Proteus & mirabilis & 15953 & IHMA & 1468358 \\
\hline 66 & P. mirabilis & Proteus & mirabilis & 15955 & IHMA & 1472077 \\
\hline 67 & P. mirabilis & Proteus & mirabilis & 15956 & IHMA & 1474630 \\
\hline 68 & P. mirabilis & Proteus & mirabilis & 15957 & IHMA & 1474632 \\
\hline 69 & P. mirabilis & Proteus & mirabilis & 15958 & IHMA & 1474633 \\
\hline 70 & P. mirabilis & Proteus & mirabilis & 15959 & IHMA & 1474635 \\
\hline
\end{tabular}




\begin{tabular}{|c|c|c|c|c|c|c|}
\hline 71 & P. mirabilis & Proteus & mirabilis & 15969 & IHMA & 1480091 \\
\hline 72 & P. mirabilis & Proteus & mirabilis & 15971 & IHMA & 1480854 \\
\hline 73 & P. mirabilis & Proteus & mirabilis & 15974 & IHMA & 1487065 \\
\hline 74 & P. mirabilis & Proteus & mirabilis & 15976 & IHMA & 1488818 \\
\hline 75 & P. mirabilis & Proteus & mirabilis & 15980 & IHMA & 1490794 \\
\hline 76 & P. mirabilis & Proteus & mirabilis & 15982 & IHMA & 1495088 \\
\hline 77 & P. mirabilis & Proteus & mirabilis & 15983 & IHMA & 1502874 \\
\hline 78 & P. mirabilis & Proteus & mirabilis & 15986 & IHMA & 1503313 \\
\hline 79 & P. mirabilis & Proteus & mirabilis & 15987 & IHMA & 1503315 \\
\hline 80 & P. mirabilis & Proteus & mirabilis & 15988 & IHMA & 1521541 \\
\hline 81 & P. mirabilis & Proteus & mirabilis & 15990 & IHMA & 1542527 \\
\hline 82 & P. mirabilis & Proteus & mirabilis & 16304 & IHMA & 1487065 \\
\hline 83 & P. mirabilis & Proteus & mirabilis & 16325 & $\mathrm{BEI}$ & HМ-752 \\
\hline 84 & P. mirabilis & Proteus & mirabilis & 17393 & $\mathrm{CDC}$ & AR-0029 \\
\hline 85 & P. mirabilis & Proteus & mirabilis & 17437 & $\mathrm{CDC}$ & AR-0155 \\
\hline 86 & P. mirabilis & Proteus & mirabilis & 17440 & $\mathrm{CDC}$ & AR-0159 \\
\hline 1 & A. baumannii & Acinetobacter & baumannii & 16421 & IHMA & 902318 \\
\hline 2 & A. baumannii & Acinetobacter & baumannii & Abi0003 & IHMA & 859292 \\
\hline 3 & A. baumannii & Acinetobacter & baumannii & 17188 & IHMA & 901771 \\
\hline 4 & A. baumannii & Acinetobacter & baumannii & Abi0006 & IHMA & 967641 \\
\hline 5 & A. baumannii & Acinetobacter & baumannii & Abi0007 & IHMA & 966904 \\
\hline 6 & A. baumannii & Acinetobacter & baumannii & 16422 & IHMA & 1001776 \\
\hline 7 & A. baumannii & Acinetobacter & baumannii & Abi0011 & IHMA & 1011395 \\
\hline 8 & A. baumannii & Acinetobacter & baumannii & Abi0012 & IHMA & 1022097 \\
\hline 9 & A. baumannii & Acinetobacter & baumannii & Abi0016 & IHMA & 967373 \\
\hline 10 & A. baumannii & Acinetobacter & baumannii & Abi0018 & IHMA & 975434 \\
\hline 11 & A. baumannii & Acinetobacter & baumannii & 17189 & IHMA & 966285 \\
\hline 12 & A. baumannii & Acinetobacter & baumannii & 11490 & ATCC & 19606 \\
\hline 13 & A. baumannii & Acinetobacter & baumannii & Abi0022 & ATCC & BAA-2093 \\
\hline 14 & A. baumannii & Acinetobacter & baumannii & 17190 & IHMA & 921325 \\
\hline 15 & A. baumannii & Acinetobacter & baumannii & 17191 & IHMA & 919655 \\
\hline
\end{tabular}




\begin{tabular}{|c|c|c|c|c|c|c|}
\hline 16 & A. baumannii & Acinetobacter & baumannii & Abi0027 & IHMA & 969614 \\
\hline 17 & A. baumannii & Acinetobacter & baumannii & 17192 & IHMA & 963263 \\
\hline \multirow[t]{2}{*}{18} & A. baumannii & Acinetobacter & baumannii/nos & Abi0033 & & \\
\hline & & & ocomialis & & IHMA & 957497 \\
\hline 19 & A. baumannii & Acinetobacter & baumannii & Abi0037 & IHMA & 993724 \\
\hline 20 & A. baumannii & Acinetobacter & baumannii & Abi0048 & IHMA & 954305 \\
\hline 21 & A. baumannii & Acinetobacter & baumannii & Abi0049 & IHMA & 925134 \\
\hline \multirow[t]{2}{*}{22} & A. baumannii & Acinetobacter & baumannii/nos & Abi0051 & & \\
\hline & & & ocomialis & & IHMA & 926001 \\
\hline 23 & A. baumannii & Acinetobacter & baumannii & 17407 & $\mathrm{CDC}$ & AR-0063 \\
\hline 24 & A. baumannii & Acinetobacter & baumannii & Abi0166 & IHMA & 976489 \\
\hline 25 & A. baumannii & Acinetobacter & baumannii & Abi0167 & IHMA & 976491 \\
\hline 26 & A. baumannii & Acinetobacter & baumannii & Abi0168 & IHMA & 976492 \\
\hline 27 & A. baumannii & Acinetobacter & baumannii & Abi0169 & IHMA & 976493 \\
\hline 28 & A. baumannii & Acinetobacter & baumannii & Abi0170 & IHMA & 969522 \\
\hline 29 & A. baumannii & Acinetobacter & baumannii & 17195 & IHMA & 969543 \\
\hline 30 & A. baumannii & Acinetobacter & baumannii & Abi0172 & IHMA & 969538 \\
\hline 31 & A. baumannii & Acinetobacter & baumannii & Abi0173 & IHMA & 953537 \\
\hline 32 & A. baumannii & Acinetobacter & baumannii & Abi0174 & IHMA & 954270 \\
\hline 33 & A. baumannii & Acinetobacter & baumannii & Abi0175 & IHMA & 954307 \\
\hline 34 & A. baumannii & Acinetobacter & baumannii & Abi0176 & IHMA & 954315 \\
\hline 35 & A. baumannii & Acinetobacter & baumannii & Abi0177 & IHMA & 954316 \\
\hline 36 & A. baumannii & Acinetobacter & baumannii & Abi0179 & IHMA & 933176 \\
\hline 37 & A. baumannii & Acinetobacter & baumannii & Abi0180 & IHMA & 982276 \\
\hline 38 & A. baumannii & Acinetobacter & baumannii & 17197 & IHMA & 969576 \\
\hline 39 & A. baumannii & Acinetobacter & baumannii & 17198 & IHMA & 946011 \\
\hline 40 & A. baumannii & Acinetobacter & baumannii & Abi0195 & IHMA & 1046662 \\
\hline 41 & A. baumannii & Acinetobacter & baumannii & Abi0196 & IHMA & 1046726 \\
\hline 42 & A. baumannii & Acinetobacter & baumannii & Abi0197 & IHMA & 1046729 \\
\hline 43 & A. baumannii & Acinetobacter & baumannii & Abi0198 & IHMA & 1046730 \\
\hline 44 & A. baumannii & Acinetobacter & baumannii & Abi0199 & IHMA & 1006472 \\
\hline
\end{tabular}




\begin{tabular}{|c|c|c|c|c|c|c|}
\hline 45 & A. baumannii & Acinetobacter & baumannii & Abi0200 & IHMA & 1006481 \\
\hline 46 & A. baumannii & Acinetobacter & baumannii & Abi0203 & IHMA & 1036387 \\
\hline 47 & A. baumannii & Acinetobacter & baumannii & Abi0204 & IHMA & 1036560 \\
\hline 48 & A. baumannii & Acinetobacter & baumannii & Abi0205 & IHMA & 1017294 \\
\hline 49 & A. baumannii & Acinetobacter & baumannii & Abi0208 & IHMA & 1093909 \\
\hline 50 & A. baumannii & Acinetobacter & baumannii & Abi0209 & IHMA & 1072181 \\
\hline 51 & A. baumannii & Acinetobacter & baumannii & Abi0212 & IHMA & 1064533 \\
\hline 52 & A. baumannii & Acinetobacter & baumannii & Abi0214 & IHMA & 1065041 \\
\hline 53 & A. baumannii & Acinetobacter & baumannii & Abi0297 & IHMA & 1231332 \\
\hline 54 & A. baumannii & Acinetobacter & baumannii & Abi0298 & IHMA & 1247256 \\
\hline 55 & A. baumannii & Acinetobacter & baumannii & Abi0305 & IHMA & 1239348 \\
\hline 56 & A. baumannii & Acinetobacter & baumannii & Abi0309 & IHMA & 1274612 \\
\hline 57 & A. baumannii & Acinetobacter & baumannii & & IHMA & 1284656 \\
\hline 58 & A. baumannii & Acinetobacter & baumannii & Abi0311 & IHMA & 1284654 \\
\hline 59 & A. baumannii & Acinetobacter & $\begin{array}{c}\text { baumannii/nos } \\
\text { ocomialis }\end{array}$ & Abi0350 & IHMA & 1202003 \\
\hline 60 & A. baumannii & Acinetobacter & baumannii & Abi0351 & IHMA & 1289610 \\
\hline 61 & A. baumannii & Acinetobacter & baumannii & Abi0352 & IHMA & 1239593 \\
\hline 62 & A. baumannii & Acinetobacter & baumannii & Abi0353 & IHMA & 1297654 \\
\hline 63 & A. baumannii & Acinetobacter & baumannii & Abi0354 & IHMA & 1275709 \\
\hline 64 & A. baumannii & Acinetobacter & baumannii & Abi0356 & IHMA & 1267976 \\
\hline 65 & A. baumannii & Acinetobacter & baumannii & Abi0380 & IHMA & 1383209 \\
\hline 66 & A. baumannii & Acinetobacter & baumannii & Abi0381 & IHMA & 1406947 \\
\hline 67 & A. baumannii & Acinetobacter & baumannii & Abi0382 & IHMA & 1406950 \\
\hline 68 & A. baumannii & Acinetobacter & baumannii & Abi0383 & IHMA & 1411372 \\
\hline 69 & A. baumannii & Acinetobacter & $\begin{array}{c}\text { baumannii/nos } \\
\text { ocomialis }\end{array}$ & Abi0384 & IHMA & 1411588 \\
\hline 70 & A. baumannii & Acinetobacter & baumannii & Abi0385 & IHMA & 1412393 \\
\hline 71 & A. baumannii & Acinetobacter & baumannii & Abi0386 & IHMA & 1412398 \\
\hline 72 & A. baumannii & Acinetobacter & baumannii & Abi0387 & IHMA & 1412675 \\
\hline 1 & P. aeruginosa & Pseudomonas & aeruginosa & Pae0001 & IHMA & 896425 \\
\hline
\end{tabular}


Pae0002

IHMA

889056

Pseudomonas

aeruginosa

17204

IHMA

860804

Pseudomonas

aeruginosa

Pae0004

IHMA

862369

Pseudomonas

aeruginosa

16654

IHMA

893827

udomonas

aeruginosa

16655

IHMA

951045

Pseudomonas

Pseudomonas

Pseudomonas

Pseudomonas

Pseudomonas

Pseudomonas

Pseudomonas

Pseudomonas

Pseudomonas

Pseudomonas

Pseudomonas

Pseudomonas

Pseudomonas

Pseudomonas

Pseudomonas

Pseudomonas

Pseudomonas

Pseudomonas

Pseudomonas

Pseudomonas

Pseudomonas

Pseudomonas

Pseudomonas

Pseudomonas

Pseudomonas

IHMA

1038443

aeruginosa

aeruginosa

aeruginosa

aeruginosa

IHMA

1018061

1007746

Pae0012 IHMA 991480

Pae0013 IHMA 996112

aeruginosa

aeruginosa

aeruginosa

aeruginosa

aeruginosa

aeruginosa

aeruginosa

aeruginosa

aeruginosa

aeruginosa

aeruginosa

aeruginosa

aeruginosa

aeruginosa

aeruginosa

aeruginosa

aeruginosa

aeruginosa

aeruginosa

aeruginosa

aeruginosa

Pae0016 IHMA

948787

987399

25102

27853

10145

47085

BAA-47

17206

958640

951048

943049

920999

941000

961209

920572

960650

971750

993862

$\begin{array}{lll}\text { Pae0046 } & \text { IHMA } & 984821 \\ \text { Pae0047 } & \text { IHMA } & 924347\end{array}$

$\begin{array}{lll}\text { Pae0048 IHMA } & 1022450\end{array}$ 


\begin{tabular}{|c|c|c|c|c|c|c|}
\hline 33 & P. aeruginosa & Pseudomonas & aeruginosa & Pae0049 & IHMA & 949064 \\
\hline 34 & P. aeruginosa & Pseudomonas & aeruginosa & Pae0050 & IHMA & 1032724 \\
\hline 35 & P. aeruginosa & Pseudomonas & aeruginosa & Pae0051 & IHMA & 943322 \\
\hline 36 & P. aeruginosa & Pseudomonas & aeruginosa & Pae0054 & IHMA & 1038439 \\
\hline 37 & P. aeruginosa & Pseudomonas & aeruginosa & Pae0055 & IHMA & 971154 \\
\hline 38 & P. aeruginosa & Pseudomonas & aeruginosa & 17209 & IHMA & 974860 \\
\hline 39 & P. aeruginosa & Pseudomonas & aeruginosa & Pae0057 & IHMA & 937137 \\
\hline 40 & P. aeruginosa & Pseudomonas & aeruginosa & Pae0058 & IHMA & 996435 \\
\hline 41 & P. aeruginosa & Pseudomonas & aeruginosa & Pae0059 & IHMA & 1018062 \\
\hline 42 & P. aeruginosa & Pseudomonas & aeruginosa & Pae0060 & IHMA & 1030837 \\
\hline 43 & P. aeruginosa & Pseudomonas & aeruginosa & Pae0061 & IHMA & 951261 \\
\hline 44 & P. aeruginosa & Pseudomonas & aeruginosa & Pae0062 & IHMA & 937446 \\
\hline 45 & P. aeruginosa & Pseudomonas & aeruginosa & Pae0063 & IHMA & 990960 \\
\hline 46 & P. aeruginosa & Pseudomonas & aeruginosa & Pae0064 & IHMA & 991711 \\
\hline 47 & P. aeruginosa & Pseudomonas & aeruginosa & Pae0065 & IHMA & 1022447 \\
\hline 48 & P. aeruginosa & Pseudomonas & aeruginosa & Pae0066 & IHMA & 985189 \\
\hline 49 & P. aeruginosa & Pseudomonas & aeruginosa & Pae0068 & IHMA & 1039336 \\
\hline 50 & P. aeruginosa & Pseudomonas & aeruginosa & Pae0069 & IHMA & 1032932 \\
\hline 51 & P. aeruginosa & Pseudomonas & aeruginosa & Pae0070 & IHMA & 976713 \\
\hline 52 & P. aeruginosa & Pseudomonas & aeruginosa & Pae0071 & IHMA & 958122 \\
\hline 53 & P. aeruginosa & Pseudomonas & aeruginosa & 16658 & IHMA & 977435 \\
\hline 54 & P. aeruginosa & Pseudomonas & aeruginosa & Pae0073 & IHMA & 939821 \\
\hline 55 & P. aeruginosa & Pseudomonas & aeruginosa & Pae0074 & IHMA & 939917 \\
\hline 56 & P. aeruginosa & Pseudomonas & aeruginosa & Pae0075 & IHMA & 1050103 \\
\hline 57 & P. aeruginosa & Pseudomonas & aeruginosa & Pae0076 & IHMA & 948761 \\
\hline 58 & P. aeruginosa & Pseudomonas & aeruginosa & 17428 & $\mathrm{CDC}$ & AR-0111 \\
\hline 59 & P. aeruginosa & Pseudomonas & aeruginosa & 17427 & $\mathrm{CDC}$ & AR-0110 \\
\hline 60 & P. aeruginosa & Pseudomonas & aeruginosa & 17426 & $\mathrm{CDC}$ & AR-0108 \\
\hline 61 & P. aeruginosa & Pseudomonas & aeruginosa & 17425 & $\mathrm{CDC}$ & AR-0103 \\
\hline 62 & P. aeruginosa & Pseudomonas & aeruginosa & 17210 & $\mathrm{CDC}$ & AR-0100 \\
\hline 63 & P. aeruginosa & Pseudomonas & aeruginosa & 17420 & $\mathrm{CDC}$ & AR-0095 \\
\hline
\end{tabular}




$\begin{array}{lllllll}64 & \text { P. aeruginosa } & \text { Pseudomonas } & \text { aeruginosa } & 17408 & \text { CDC } & \text { AR-0064 } \\ 65 & \text { P. aeruginosa } & \text { Pseudomonas } & \text { aeruginosa } & \text { Pae0102 } & \text { ATCC } & 27589 \\ 66 & \text { P. aeruginosa } & \text { Pseudomonas } & \text { aeruginosa } & \text { Pae0103 } & \text { ATCC } & 33363 \\ 67 & \text { P. aeruginosa } & \text { Pseudomonas } & \text { aeruginosa } & \text { Pae0128 } & \text { IHMA } & 1320071 \\ 68 & \text { P. aeruginosa } & \text { Pseudomonas } & \text { aeruginosa } & \text { Pae0129 } & \text { IHMA } & 1078276 \\ 69 & \text { P. aeruginosa } & \text { Pseudomonas } & \text { aeruginosa } & \text { Pae0130 } & \text { IHMA } & 1151373 \\ 70 & \text { P. aeruginosa } & \text { Pseudomonas } & \text { aeruginosa } & \text { Pae0131 } & \text { IHMA } & 1145010 \\ 71 & \text { P. aeruginosa } & \text { Pseudomonas } & \text { aeruginosa } & \text { Pae0132 } & \text { IHMA } & 1307184 \\ 72 & \text { P. aeruginosa } & \text { Pseudomonas } & \text { aeruginosa } & \text { Pae0133 } & \text { IHMA } & 1309009 \\ 73 & \text { P. aeruginosa } & \text { Pseudomonas } & \text { aeruginosa } & \text { Pae0134 } & \text { IHMA } & 1346392 \\ 74 & \text { P. aeruginosa } & \text { Pseudomonas } & \text { aeruginosa } & \text { Pae0137 } & \text { IHMA } & 1178310 \\ 75 & \text { P. aeruginosa } & \text { Pseudomonas } & \text { aeruginosa } & \text { Pae0138 } & \text { UoH } & \text { SED S2 } \\ 76 & \text { P. aeruginosa } & \text { Pseudomonas } & \text { aeruginosa } & \text { Pae0139 } & \text { UoH } & \text { SED S3 } \\ 77 & \text { P. aeruginosa } & \text { Pseudomonas } & \text { aeruginosa } & \text { Pae0140 } & \text { UoH } & \text { SED S14 } \\ 78 & \text { P. aeruginosa } & \text { Pseudomonas } & \text { aeruginosa } & \text { Pae0141 } & \text { UoH } & \text { SED S18 } \\ 79 & \text { P. aeruginosa } & \text { Pseudomonas } & \text { aeruginosa } & \text { Pae0142 } & \text { UoH } & \text { SED S30 } \\ 80 & \text { P. aeruginosa } & \text { Pseudomonas } & \text { aeruginosa } & \text { Pae0143 } & \text { UoH } & \text { PA14 } \\ 81 & \text { P. aeruginosa } & \text { Pseudomonas } & \text { aeruginosa } & \text { Pae0144 } & \text { KUL } & \text { PA01k }\end{array}$

Legend- American Type Culture Collection (ATCC), International Health Management Associates (IHMA), US Centers for Disease Control and Prevention (CDC), Biodefense and Emerging Infections Research Resources Repository (BEI), Texas Tech University Health Sciences Center (TTU), University of Nottingham (UoH), Katholieke Universiteit Leuven (KUL). 
Table S4 Strains used in CRE Proof of Concept Assay

\begin{tabular}{cccccccc}
\hline Genus & Species & Designation & RMSCC \# & Source & Source ID & $\begin{array}{c}\text { Ertapenem MIC } \\
\text { (ug/mL) }\end{array}$ & $\begin{array}{c}\text { Meropenem MIC } \\
\text { (ug/mL) }\end{array}$ \\
\hline & & & & & & & \\
Escherichia & coli & Sensitive & 15729 & In-house & 719024 & 0.4999 & 0.2499 \\
Escherichia & coli & Resistant & 15746 & In-house & 131550 & 8.0001 & 16.0001 \\
Klebsiella & pneumoniae & Sensitive & 959479 & JMI Labs & 959479 & 0.5 & 0.06 \\
Klebsiella & pneumoniae & Resistant & 1037210 & JMI Labs & 1037210 & 8.0001 & 8.0001 \\
Enterobacter & cloacae & Sensitive & 902962 & JMI Labs & 902962 & 0.06 & 0.03 \\
Enterobacter & cloacae & Resistant & 968217 & JMI Labs & 968217 & 8.0001 & 8.0001
\end{tabular}


Supplementary Amino Acid

\section{Sequences}

Underlined sequence is from $\mathrm{Tfib}_{\mathrm{P} 1 \mathrm{~s}}$ used in hybrid fusion proteins

Sequence S1 Banana Tail Fiber Fusion

$\operatorname{Tfib}_{\text {Banana }}(\mathrm{Hyb})$

MNDVTVVTSVTYPSSESLALVADVQYHEPYLSAALN RKFRGIVDPGFYAGFLPKPGGGMNLLITSVDGDKTA GAASVDIGEFYQVTIQQRKDISLALSAGKKYAIVLKGR YLLGEDTYQVNTASHIHAAEFVARTYTDSYQLGDGE LLVCTVNIPASVSAITQEMIDTSERINRSIGIDISDSVTS TRSDVAASSLAVKKAYDLAKSKYTAQDATTARKGIV QLSSATDSTSEALAATPKAVKAVNDNANGRVPSARK VNGRALASDISITAQDIFNGQAVAIGNAADLNAYTT PGLYYQPANAQAQTGRNYPEANAGSLEVYKHAGIT QIYRIYNSSRSYIRTLYSGTWSAWVKQYDAANKPSPA DINAVNKGGDTMTGGLKIRAADALRIYDAAYGMIFR RSENNFYLIPTAKDQGEDGGISGLRPLYIDLTNGRVTL GNGAVVNGGLGLGVVSGLGGNSIALGDNDTGFKQ NGDGVLDVYANSKQVMRFLNSGITSYMLFNMNAG ASVSSTLTFKNGSGITSEKTGANPRNGRIYWGGDAS RGNRIEFADDAGWKAYIERHPSNGVQLVVNGRING SIVYSSGEVLAGGGSARFAADGNIFGSKWGNQWLD AYLKNTYQPKGNYTPAGQAYTKVESDGRFQPKGSYT PAGQAYTKAESDARYNLKNTATKSANAMTHKDAST GVMEVVMSNINVPNKTNVNVTFPAAFPNACVGVVI TYNGAGHGSGDDSAIYVPSYSRTGCTLYAHNADGKF MLIAKGY*

Sequence S2 Banana tail fiber assembly protein $\mathrm{Tfa}_{\text {Banana }}$

MRIYFSPSEIGFYHESDKQAYLLAGTWPNDLLEISEK WFLYLLEGQQKGKVITVNDYDQPVLVDPPTATKEQL LAEADAQKEALMNSASAVIEPLKDAVELGMSTDEEE GLLLAWQQYRVLLMRVDTSHAPDIEWPVLPA*

Sequence S3 Gold tail fiber fusion $\mathrm{Tfib}_{\mathrm{Gold}}(\mathrm{Hyb})$

MNDVTVVTSVTYPSSESLALVADVQYHEPYLSAALN RKFRGIVDPGFYAGFLPKPGGGMNLLITSVDGDKTA GAASVDIGEFYQVTIQQRKDISLALSAGKKYAIVLKGR YLLGEDTYQVNTASHIHAAEFVARTYTDSYQLGDGE LLVCTVNIPASVSAITQEMIDTSERINRSIGIDISDSVTS
TRSDVAASSLAVKKAYDLAKSKYTAQDATLLAKGFTQ LSNDSNSGSETLAATPKAVKAVNDASLKIAANLKDLP NKSVARGNLELGTAATRNVGAQKTNLMEVGAFGL GGGPIHREDALSNRGEIYRVTGASKNAPGGGVYGVL NLPCDGGPSSGYLAIQPNGSSYIGTSTTPDKPLNWYR IYTTGFKPTATDVDAYSKAEADGKFVKQSGDTITGAL TVNGAIESKSGLTTPSLAVNGSVTIAGALTTKAGVELF GTTPYLDFHYGNSNGDFDVRLINDNKGTLAFHGNEY YVNGKLSATGDAWIGGKANINGMAAFYSSDFITKQ GNLTHPDGNRQTNGMRLQGQGNLLVDLYHYEKVG SHHEFGIHVANGGADGWFSFRNNGELRANGTLFAA GAAYQTDGNINGGIWGGYLSNYLNHNFVRDVRLG NVESIATWRGPGYSDSAGYVLTGAANNNVDEYIDVI FRRPLQKHIGGNWVTVWSV

Sequence $\mathbf{S 4}$ Gold tail fiber assembly protein $\mathrm{Tfa}_{\text {Gold }}$

MMNNMKNFTLAAPETVEQKQLAASHGVLFLKSDA GEDWYECQKSFRPETVKLMYDKDGIIRSITAKPNAE GHYDVSGFFPENMSVAEVENLPEGADINGRWIFDG ENIIPRSYSTQELRQQAANKKQELIKQSSLQIETLNDA TDLGMASEEELRLLTRWKTYRVLLNRVDPEAAPDID WPQPPQ

Sequence S5 Jazzberry tail fiber fusion

$\mathrm{Tfib}_{\text {Jazzberry }}(\mathrm{Hyb})$

MNDVTVVTSVTYPSSESLALVADVQYHEPYLSAALN RKFRGIVDPGFYAGFLPKPGGGMNLLITSVDGDKTA GAASVDIGEFYQVTIQQRKDISLALSAGKKYAIVLKGR YLLGEDTYQVNTASHIHAAEFVARTYTDSYQLGDGE LLVCTVNIPASVSAITQEMIDTSERINRSIGIDISDSVTS TRSDVAASSLAVKKAYDLAKSKYTAQDASTTQKGLV QLSSATNSDSETMAATPKAVKSIKDLADTKAPIESPSL TGTPTAPTAAQGTNSTQIANTAFVKAAITALINGAP GTLDTLKEIAAAINNDPNYSTTINNALALKAPLASPAL TGVPTAPTAAQGTNNTQIATTAYVRAAISALVGSSP EALDTLYELAAALGNDPNFATTMTNALAGKQPLDAT LTALAGLATGANKLPYFTGTDTVSQTDLTSVGRDILA KTSILAVIQYLGLRELGTSGEKIPLLSTANTWSARQTF NGGITGALTGNADTATKLKTARKINNVSFDGSADITL TPENLGVTSLTFEKNNGEMPIDADLNTFGPVEAYLG VWSKATSTNATLEKNFPEDNAVGVLEVFAAGNFAG TQRFTTRDGNVYIRRLANKWNGSDGPWGIWRHTQ SATRPLSTTIDLNTLGAAEHLGLWRNSSSAIASYERNY 
PEEGGFAQGVLEILEGGNYGRTQRYTTRRGNMYVR CLAASWDASNPQWEPWLKVGHQSESRYYEGDLNV LTDPGIYSVTGKATNGPMLDTVGATLLGILEVIRRFD GVSVWQRYTTTGKSETTQGRTFERVYAGSKWTEW REVYNSFSLPLNLGIGGAVAKLSSLDWQTYDFVPGSL ITVRLDNMTNIPDGMDWGVIDGNLINIAVGPSDDS GTGRSMHVWRSTVSKANYRFFMVRISGNPGSRTIT ARRVPIIDEAQTWGAKQTFSAGLSGELSGNAATATK LKTARKINNVSFDGSGDIEVLPVGVPLPWPSDTVPS GYALMQGQTFDKSAYPKLAAAYPSGVIPDMRGWTI KGKPASGRDVLSLEQDGIKSHTHSASASNTDLGTKTT SSFDYGTKSTNNTGAHTHNVSGTANSAGAHTHTVP LRRPNSGGMNFDWLDGASSGTVVGNGTVPSSGAH THSVSGTATSAGAHAHTVGIGAHTHSVAIGSHGHTI TVNAAGNAENTVKNIAFNYIVRLA*

Sequence $\mathbf{S 6}$ Jazzberry tail fiber assembly protein $\mathrm{Tfa}_{\text {Jazzyberry }}$

MAFRMSEQSRTVKIYNLLAGTNEFIGEGDAYIPPHT GLPANSTDIAPPEIPAGFVAVFNSENESWNIVEDHR GKTVYDVASGDALFISEPGPLPENVTWLSPAGEYQK WDGVSWVKDEEAEKLFRIREAEEKKARLIQEATDNI AILQDAVNLEIATNEENSQLDSWRKYRVLVSRIDTST APDIVWPELMNQGYVREDEQITSD*

Sequence $\mathbf{S 7}$ Orchid tail fiber fusion

$\mathrm{Tfib}_{\text {Orchid }}(\mathrm{Hyb})$

MNDVTVVTSVTYPSSESLALVADVQYHEPYLSAALN RKFRGIVDPGFYAGFLPKPGGGMNLLITSVDGDKTA GAASVDIGEFYQVTIQQRKDISLALSAGKKYAIVLKGR YLLGEDTYQVNTASHIHAAEFVARTYTDSYQLGDGE LLVCTVNIPASVSAITQEMIDTSERINRSIGIDISDSVTS TRSDVAASSLAVKKAYDLAKSKYTAQDASTTQKGLV QLSSATNSDSETMAATPKAVKSIKDLADTKAPIESPSL TGTPTAPTAAQGTNSTQIANTAFVKAAITALINGAP GTLDTLKEIAAAINNDPNYSTTINNALALKAPLASPAL TGVPTAPTAAQGTNNTQIATTAYVRAAISALVGSSP EALDTLYELAAALGNDPNFATTMTNALAGKQPLDAT LTALAGLATGANKLPYFTGTDTVSQTDLTSVGRDILA KTSILAVIQYLGLRELGTSGEKIPLLSTANTWSARQTF NGGITGALTGNADTATKLKTAARSIGGVAFDGSANI NLPGVNTTGNQNTTGNAATATKLATARNINGVKFD GSVDISIPTITSRGRVTALTGTTQGAATGLQMYEAYN NGYPSAYGNVLHLKGATAVGEGELFIGWSGTSGAH
APVHVRSRRDTDTASWSEWAQVYTSKDSIPGVNTT GNQNTTGNAASATKLQTARTIGGVSFNGTANIDLP GVNKTGNQNTTGNAATATKLQTARTINGVSFDGSK NIELTAEDLNLQEFINKANNAVQRSGDTLSGGLTFKN DSILAWIRNTDWAKIGFKNDADGDTDSYMWFETG DNGNEYFKWRSKQSTTTKDLMNLKWDALYVLVNAI VNGEVISKSANGLRIAYGNYGFFIRNDGSDTYFMLT NSGDNMGTYNGLRPLWINNATGAVSMGRGLNVS GETLSDRFAINSSTGMWINMRDQNVIMGRNAVST DGAQALLRQDHADRKFMIGGLGNKQFGIYMINNSR TANGTDGQAYMDNNGNWLCGAQIIPGNYGNFDS RYVKDVRLGTRVVQLMARGGRYEKAGHAITGLRIIG EVDGDDEAIFRPIQKYINGTWYNVAQV

Sequence $\mathbf{5 8}$ Orchid tail fiber assembly protein Tfa Orchid

MQHLKNIRSGNPKTKEQYQLTKNFDVIWLWSEDGK NWYEEVKNFQPDTIKIVYDENNIIVAITKDASTLNPE GFSVVEVPDITANRRADDSGKWMFKDGAVVKRIYT ADEQQQQAESQKAALLSEAESVIQPLERAVRLNMA TDEERTRLEAWERYSVLVSRVDTANPEWPQKPE

Sequence $\mathbf{S 9}$ Rio tail fiber fusion $\mathrm{Tfib}_{\mathrm{Rio}}(\mathrm{Hyb})$

MNDVTVVTSVTYPSSESLALVADVQYHEPYLSAALN RKFRGIVDPGFYAGFLPKPGGGMNLLITSVDGDKTA GAASVDIGEFYQVTIQQRKDISLALSAGKKYAIVLKGR YLLGEDTYQVNTASHIHAAEFVARTYTDSYQLGDGE LLVCTVNIPASVSAITQEMIDTSERINRSIGIDISDSVTS TRSDVAASSLAVKKAYDLAKSKYTAQDATTTVKGFTK LNNQVGDSENTALTPYGAKKELENYQPKGNYQPAG NYVTTATFNLEINKKIDKASLSQQLGNDVNKVPSLDL VTKELGNKQPKGDYALKTWVDNIYYKRDDSPSFNEI SATAIRVNSVNVALKGDCYTKSESDNKYSPKLPFVYS SGEESKIISPNNKILIFVNDNGTTGGYDRDTQATVWG FDKRGFMTRGTVHFDRMSGVYSQSQVDAKLKECLN LKTSSKQFIASDIYAPTFITGSQYGERSYLEHKSDELTIT HISPKSGATNITCRNKSGTLALLGDLEDVNNIPVGSPI PWSLATAPSGYLICNGQTFNKSTYPKLAVAYPSGKLP DLRGEFIRGLDSGRGIDAGRSVLSVQKGNSLLSSNIF GGLSSSESNKWHKAINYIGITGTDNDGGYGVHQQIE GANGNETRPRNIAFLYIVRAA

Sequence S10 Rio tail fiber assembly protein $\mathrm{Tfa}_{\text {Rio }}$ 
MSKYSLDIQKGKIGDNGLAEIAGWVKCYLAHPITHEY MGATMENVMFDVSLSAGAYLDEPPLPQKENQAVR RREDGSAWEIVDDCRGLTAYNTQTKQPIIIDFMGPL PESLTLLKPNSEFDKWDGKNWIVDTEAQKAALITQIK QEKSRRLDEADNIITYLQEAVDVDLATEEEATALQK WKKYRVLLNRVDISTAPDIEWPEKPQ

Sequence S11 Shamrock tail fiber fusion Tfib $_{\text {sfMus' }}(\mathrm{Hyb})$

MNDVTVVTSVTYPSSESLALVADVQYHEPYLSAALN RKFRGIVDPGFYAGFLPKPGGGMNLLITSVDGDKTA GAASVDIGEFYQVTIQQRKDISLALSAGKKYAIVLKGR YLLGEDTYQVNTASHIHAAEFVARTYTDSYQLGDGE LLVCTVNIPASVSAITQEMIDTSERINRSIGIDISDSVTS TRSDVAASSLAVKKAYDLAKSKYTAQDASTTQKGLV QLSSATNSDSETMAATPKAVKSIKDLADTKAPIESPSL TGTPTAPTAAQGTNSTQIANTAFVKAAITALINGAP GTLDTLKEIAAAINNDPNYSTTINNALALKAPLASPAL TGVPTAPTAAQGTNNTQIATTAYVRAAISALVGSSP EALDTLYELAAALGNDPNFATTMTNALAGKQPLDAT LTALAGLATGANKLPYFTGTDTVSQTDLTSVGRDILA KTSILAVIQYLGLRELGTSGEKIPLLSTANTWSARQTF NGGITGALTGNADTATKLKTARNINGVRFDGSGDIN INTLVSRGRVTALEANAQGTSGIQLYEAYNNGYPSPY GNVLHLKGATAAGEGELFIGWSGTSGAHAPVHIRSR RDTDSANWSEWAQVYTSKDSIPGVNAKGDQDTSG NAATATKLQTACTINGVSFDGSKNIELTAEDLNLQET VNKADNAVQKTGDTLSGGLTFENDSILAWIRNTDW AKIGFKNNSDADTDSYMWFETGNNGNEYFKWRHR IIGTRPKDLMNLKWNALSVLVEALFSSEVKISTVNALR IFNSSFGAIFRRSEECLHIIPTRENEGENGNIGPLRPFT LNLRTGRISMGHGLDVTGDIFANRFAINSSTGMWIH MRDQNVILGRNAVSTDGAQALLRQDHADRKFMIG GLGNKQFGIYMINNSRTANGTDGQAYMDNNGNW LCGAQVIPGNYGNFDSRYVKDVRLGSQQYYGVNN WQTWNFQCPSGHVLSGINVQDTGKNSADNIAGVY YRPVQKYINGTWYNVASV*

Sequence $\mathbf{S 1 2}$ Shamrock tail fiber assembly protein $\mathrm{Tfa}_{\text {sfMus' }}$

MMHLKNIKAGNAKTLEQYELTKKHGVIWLYAEDGK NWYEEVKNFQPDTIKIVYDENNIIVAVTKDASTLNPE GFSVVEIPDITANRRADDSGKWMFKDGAVVRRTYT
ADEQQQQAESQKAALLSEAESVIQPLERAVRLNMA TDEEHARLESWERYSVLVSRVDTANPEWPQKPE*

Sequence S13 Tangerine tail fiber Tfib p15BSvo $_{\text {p }}$ MNDVTVVTSVTYPSPESLALVADVQYHEPYLSAALN RKFRGIVDPGFYAGFLPKPGGGMNLLITSVDGDKTA GAASVDIGEFYQVTIQHRKDISLALNAGKKYAIVLKG RYLLGEDTYQVNTASHIHAAEFVARTYTDSYQLGDG ELLVCTVNIPAGVSTITQEMIDTSERINRTIGIDISDSV TSTRSDVAASSLAVKKAYDLAKSKYTAQDASTTQKGL VQLSSATNSTSEVLAATPKAVKAAYDLANGKYTAQD ATTTQKGIVQLSSDTNSTSETLAATPKAVKAAYDLAA GKAPSSHTHPWNQITGVPTASLTAKGITQLSSATNS TSEVLAATPKAVKAAYDLANGKYTAQDATTAQKGIV QLSSATNSTSEVLAATPKAVKAAYDLANGKYTAQDA TTTQKGIVQLSSDTNSTSETLAATPKAVKAAYDLAAG KAPSSHTHPWNQITVVPTASLTAKGITQLSSATNSTS EVLAATPKAVKAAYDLANGKYTAQDATTAQKGIVQL SSATNSTSEVLAATPKAVKAAYDLANGKQAADATLT ALAALATAADKLPYFTGVDRAALTALTSVGRAILGKT SIQSVLDYLGLGEGSALPVGVPVPWPSATPPTGWLK CNGAAFSSEKYPNLAKVYPTLKLPDLRGEFIRGWDDS RGIDTGRSLLSGQTATFIRTALQDYYGVDLTTNVKVG IAYATADSVITVGNPANPKAGDNSDYVPASSDNSIT GTQRTAEDNFTGAWISMRPRNVAFNYIVRAT*

Sequence S14 Tangerine tail fiber assembly protein $\mathrm{Tfa}_{\mathrm{p} 15 \mathrm{BS} v \mathrm{O}}$

MDNAILNSELIAIQAGNIIVYNYDGGNREYISASTEYL AVGVGIPANSCLDAPGSHKAGYAILRSEDLSSWEYV PDHRGETVYSIDTGNPEEITVLGDYPENTTTIAPLTPY DKWDGEKWVVDTEAQHSAAVEAAETKRQSLIDTA MDSISLIQLKLRAGRKLTQAETTQLNSVLDYIDELNA MDLTTAPDLNWPEKQLSTAS* 